\title{
Search for Dark Matter and Supersymmetry with a Compressed Mass Spectrum in the Vector Boson Fusion Topology in Proton-Proton Collisions at $\sqrt{s}=8 \mathrm{TeV}$
}

\author{
V. Khachatryan et al. \\ (CMS Collaboration)
}

(Received 30 May 2016; published 12 January 2017)

\begin{abstract}
A first search for pair production of dark matter candidates through vector boson fusion in proton-proton collisions at $\sqrt{s}=8 \mathrm{TeV}$ is performed with the CMS detector. The vector boson fusion topology enhances missing transverse momentum, providing a way to probe supersymmetry, even in the case of a compressed mass spectrum. The data sample corresponds to an integrated luminosity of $18.5 \mathrm{fb}^{-1}$, recorded by the CMS experiment. The observed dijet mass spectrum is consistent with the standard model expectation. In an effective field theory, dark matter masses are explored as a function of contact interaction strength. The most stringent limit on bottom squark production with mass below $315 \mathrm{GeV}$ is also reported, assuming a $5 \mathrm{GeV}$ mass difference with respect to the lightest neutralino.
\end{abstract}

DOI: 10.1103/PhysRevLett.118.021802

Cosmological measurements indicate that dark matter (DM) constitutes $85 \%$ of all matter in the Universe [1]. The identity of DM is one of the most fundamental open questions in both particle physics and cosmology. Many extensions of the standard model (SM) predict a DM candidate in the form of a weakly interacting massive particle (WIMP) at the electroweak symmetry breaking scale.

Previously, searches for WIMP DM at the CERN LHC have been performed in the context of complete renormalizable theories, such as supersymmetry (SUSY). For example, many searches for the lightest SUSY particle (LSP) in $R$-parity-conserving SUSY [2,3] rely on production through decays of heavier particles (e.g., squarks), accessible at the LHC that gives rise to signatures with energetic leptons, photons, and/or jets. Such searches [4-7] have limited sensitivity in scenarios with a compressed mass spectrum, which results in visible particles with too little energy to be detected efficiently.

This Letter describes the first search for direct pair production of DM through pure electroweak vector boson fusion (VBF) processes at a hadron collider. The VBF production mechanism provides a probe of DM that is agnostic to the accessibility of heavier-colored or electroweak sectors. In order to study DM-SM interactions with minimal assumptions, we consider an effective field theory (EFT) approach, which provides complementary information to other DM searches [8-11]. The benchmark model

*Full author list given at the end of the article.

Published by the American Physical Society under the terms of the Creative Commons Attribution 4.0 International license. Further distribution of this work must maintain attribution to the author(s) and the published article's title, journal citation, and DOI. used assumes the DM particle to be a Dirac fermion and its interaction with the electroweak gauge bosons to be mediated by a heavy particle (dimension 5 a operator as in Ref. [12]). The EFT framework is examined with a contact interaction of scale $\Lambda=\mathcal{M} / g_{\text {eff }}=\mathcal{M} / \sqrt{g_{\chi} g_{V}}$, where $\mathcal{M}$ is the mass of the heavy mediator, $g_{\chi}$ is its coupling to the DM particle, and $g_{V}$ is its coupling to vector bosons $V=\gamma, Z$, or $W$ [Fig. 1 (left)].

The EFT benchmark model can be used to compare the results in this Letter to other analyses considering $V$ - $V$-DM-DM contact interactions, but it cannot be directly compared to searches which probe quark-DM interactions (e.g., in the monojet topology [13-15]). To demonstrate the effectiveness of this VBF analysis strategy relative to the monojet searches, we consider as a benchmark the strong production of squarks, which can satisfy the VBF selection when produced in association with two jets arising from initial-state radiation. Under the assumption that the squark and the LSP are nearly mass degenerate, the jets produced in the squark decays are typically too soft to be observed. Here, we consider bottom squarks [Fig. 1 (right)], and assume a $5 \mathrm{GeV}$ mass difference with the LSP, where the
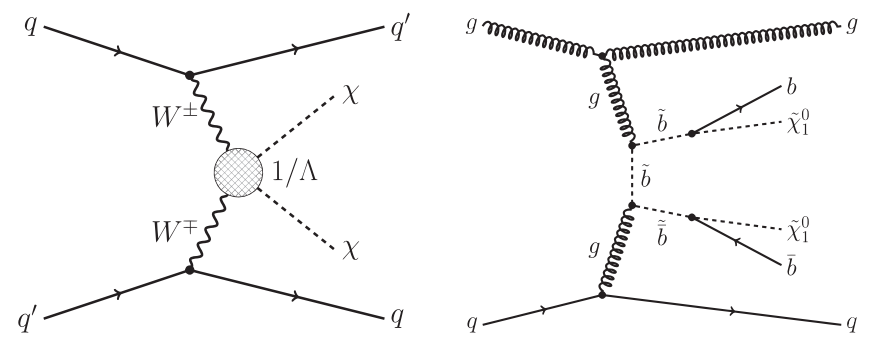

FIG. 1. Feynman diagrams for dark matter pair production in a vector boson fusion process (left) and for bottom squark pair production (right). Given a nearly degenerate bottom squark and LSP, the final-state $b$ quarks are too soft to be observed. 
monojet analyses by ATLAS and CMS [13-15] exclude masses below $\approx 250 \mathrm{GeV}$, but the analysis is applicable to all generations of squarks.

The analysis is performed using data collected with the CMS experiment at the LHC in proton-proton $(p p)$ collisions at a center-of-mass energy of $8 \mathrm{TeV}$. The data sample corresponds to an integrated luminosity of $18.5 \mathrm{fb}^{-1}$. The VBF topology is characterized by the presence of two forward jets (i.e., jets near the beam axis) in opposite hemispheres, leading to a large dijet invariant mass [16-21]. The two jets boost the decay products of new particles, similar to requiring a jet from initial state radiation, which aids event selection and enhances rejection of multijet background. We analyze the dijet mass spectrum to search for new physics in events consistent with the VBF topology and with missing transverse momentum $\left(p_{T}^{\text {miss }}\right)$.

The central feature of the CMS apparatus [22] is a superconducting solenoid of $6 \mathrm{~m}$ internal diameter, providing a magnetic field of $3.8 \mathrm{~T}$. Within the solenoid volume are a silicon pixel and strip tracker, a lead tungstate crystal electromagnetic calorimeter (ECAL), and a brass and scintillator hadron calorimeter, each composed of a barrel and two end cap sections. Forward calorimeters extend the pseudorapidity $(\eta)$ coverage provided by the barrel and end cap detectors up to $|\eta|<5$.2. Muons are measured in gasionization detectors embedded in the steel flux-return yoke outside the solenoid. A more detailed description of the CMS detector, together with a definition of the coordinate system used (including the azimuthal angle $\phi$ ), and the relevant kinematic variables can be found in Ref. [22].

The data sample was collected using an online event selection requiring events with $p_{T}^{\text {miss }}>65 \mathrm{GeV}$ and at least two jets with $p_{T}>35 \mathrm{GeV}$, with a VBF topology. This online selection has an efficiency of more than $98 \%$ for the analysis.

For the offline analysis, the events are reconstructed from particle candidates found by the particle-flow (PF) algorithm $[23,24]$, which uses reconstructed objects in an event to build candidate muons, electrons, photons, and charged and neutral hadrons. The anti- $k_{\mathrm{T}}$ algorithm [25], with a distance parameter of 0.5 , is used for jet clustering. Jets are required to pass identification criteria designed to reject particles from other interactions in the same bunch crossing (pileup) and spurious energy measurements in the calorimeters. For jets with $p_{T}>30 \mathrm{GeV}$ and $|\eta|<2.5(>2.5$ ), the identification efficiency is about 99\% (95\%), with 90-95\% (60\%) of pileup jets rejected [26]. Jets originating from the hadronization of bottom quarks are tagged using the combined secondary vertex algorithm [27,28]. For $b$-tagged jets with $p_{T}>20 \mathrm{GeV}$, the identification efficiency is $\approx 85 \%$, with a $\approx 10 \%$ (20\%) misidentification probability for light quarks and gluons (charm quarks) [28]. The electron momentum is estimated by combining the energy measurement in the ECAL with the momentum measurement in the tracker [29]. Muons are identified as a track in the central tracker, consistent with either a track or several measurements in the muon system, associated with an energy deficit in the calorimeters [30]. Taus are reconstructed using the hadron plus strips algorithm [31].

We require exactly two jets with $p_{T}>50 \mathrm{GeV}$ and $|\eta|<$ 5 in a VBF configuration, which corresponds to jets in opposite hemispheres $\left(\eta_{1} \eta_{2}<0\right)$, with large separation in pseudorapidity $(|\Delta \eta|>4.2)$, and large dijet mass $\left(m_{\mathrm{jj}}>750 \mathrm{GeV}\right)$. Events with additional jets of $p_{T}>$ $30 \mathrm{GeV}$ (jet veto) or $b$-tagged jets of $p_{T}>20 \mathrm{GeV}$ are rejected. Since there are no bottom quarks in Fig. 1 (left), and the bottom quarks in Fig. 1 (right) are too soft to identify efficiently, the rejection of events which contain a $b$-tagged jet with $p_{T}>20 \mathrm{GeV}$ is optimized to maintain high signal efficiency while reducing $t \bar{t}$ and single-top backgrounds to negligible levels. Similarly, events with isolated leptons of $p_{T}>10 \mathrm{GeV}(>15 \mathrm{GeV}$ for tau leptons) and $|\eta|<2.5$ are rejected. For electrons and muons, we define the isolation variable as the $p_{\mathrm{T}}$ sum of the reconstructed PF charged and neutral particles within a cone of radius $\Delta R=\sqrt{(\Delta \eta)^{2}+(\Delta \phi)^{2}}=0.3$, centered around the electron or muon track. We require that this isolation variable divided by the lepton's $p_{T}$ be less than 0.20 . Isolation for tau candidates is imposed by applying a dedicated multivariate discriminator, which combines the surrounding energy deposits with the median energy density flow in the event. The analysis selects events with $p_{T}^{\text {miss }}>250 \mathrm{GeV}$. To reduce contributions from jet mismeasurements, an azimuthal separation between the subleading jet and the direction of the missing transverse momentum vector, $\mid \Delta \phi\left(\vec{p}_{T}^{\text {miss }}\right.$, jet 2$) \mid>0.5$, is required. This set of requirements defines the signal region.

After this selection, the main SM contributions are from the production of $Z(\rightarrow \nu \bar{\nu})+$ jets and $W(\rightarrow \ell \nu)+$ jets (where $\ell=e, \mu, \tau$ ), with smaller contributions from QCD multijet, $t \bar{t}$, and diboson production. The $Z(\rightarrow \nu \bar{\nu})+$ jets background has the same topology as the DM or LSP signals, and is therefore mostly irreducible. Because of contribution to $p_{T}^{\text {miss }}$ from neutrinos, $W(\rightarrow \ell \nu)+$ jets events can enter the signal region if the accompanying charged lepton fails the lepton veto criteria.

Background samples for $Z(\rightarrow \nu \bar{\nu})+$ jets, $W(\rightarrow \ell \nu)+$ jets, $t \bar{t}$, and diboson production are generated with MADGRAPH (v5.1.3) [32]. Events with a Higgs boson produced through VBF are generated with POWHEG (v1.0r1380) [33,34]. Signal samples, DM pair production $(\chi \chi \mathrm{jj})$, and bottom squark pair production $(\tilde{b} \overline{\tilde{b}} \mathrm{jj})$ are generated with MADGRAPH (v5.1.5). The momentum distribution of the partons is taken from CTEQ6L1 (MADGRAPH) and CTEQ6M (POWHEG) [35], except for the VBF Higgs boson samples where CT10 [36] is used. The parton showering, fragmentation, hadronization, and various decays are performed with PYTHIA (v6.4.22) [37]. For background samples, the response of the CMS 
apparatus is simulated using GEANT4 (v9.4p03) [38], while for the signal samples, a fast simulation program [39] is used. The signal acceptance and dijet mass distribution are cross checked with the GEANT4-based simulation, and the acceptance is corrected for the small differences $(<5 \%)$ observed. To simulate the effect of pileup, additional $p p$ collisions with the multiplicity distribution matching that in data are superimposed on the hard-scattering event. Event yields are normalized to the integrated luminosity of the collision data using next-to-next-to-leading order cross section calculations, except in the case of signal samples for which next-to-leading order $(\tilde{b} \overline{\tilde{b}} \mathrm{jj})[40]$ and leading order $(\chi \chi j j)$ cross sections [32] are used.

The strategy for the background estimation is to use Monte Carlo (MC) simulations to model the $p_{T}^{\text {miss }}$ distributions, and jet and lepton vetoes. The background yields predicted by the MC simulations are corrected for observed differences, with respect to the data in control regions, and scaled to the fraction of events passing the VBF topology selection, derived from data. The modeling of the dijet mass distribution is checked in the control regions. For the $Z(\rightarrow \nu \bar{\nu})+$ jets background, we use three control regions to verify the MC simulation, estimate acceptance corrections used to scale the MC yields, and measure the fraction of events passing the VBF topology selection. The control regions are defined by treating muons as neutrinos in the $Z \rightarrow \mu^{+} \mu^{-}$decay mode. The first control region $\left(\mathrm{CR}_{Z 1}\right)$ is a $Z\left(\rightarrow \mu^{+} \mu^{-}\right)+$two jets sample, used to validate modeling of geometric and kinematic acceptance of leptons. We find a data-to-MC correction of $0.98 \pm 0.01$ (stat). For the $\mathrm{CR}_{Z 2}$ control region, which is a subset of $\mathrm{CR}_{Z 1}$, we treat the two muons as neutrinos, subtract the muon $p_{T}$ vectors from $\vec{p}_{T}^{\text {miss }}$, and require $p_{T}^{\text {miss }}>250 \mathrm{GeV}$ together with a veto on $b$-tagged jets and additional leptons, as in the analysis selection. We measure a data-to-MC correction factor of $0.95 \pm 0.06$ (stat). For $\mathrm{CR}_{Z 2}$, the non- $Z\left(\rightarrow \mu^{+} \mu^{-}\right)$contributions, about $4 \%$, are treated as an uncertainty. Adding the $\mathrm{VBF}$ topology selection defines $\mathrm{CR}_{\mathrm{Z3}}$. The ratio of $\mathrm{CR}_{\mathrm{Z3}}$ to $\mathrm{CR}_{Z 2}$ events in the data gives the fraction of $Z(\rightarrow \nu \bar{\nu})+$ jets events passing the VBF topology selection. Table I details the contributions of the major backgrounds.
To determine the contribution of $W(\rightarrow \ell \nu)+$ jets background to the analysis, we use a similar procedure. We substitute the lepton veto with a one muon requirement to obtain a $W(\rightarrow \mu \nu)$ plus two jets sample, $\mathrm{CR}_{W 1}$. The data-to$\mathrm{MC}$ correction factor for the sample is $0.97 \pm 0.01$ (stat). Treating the muon as undetected and requiring $p_{T}^{\text {miss }}>250 \mathrm{GeV}$, and the veto on $b$-tagged jets and additional leptons, as in the analysis selection, defines $\mathrm{CR}_{W 2}$. We measure a data-to-MC correction factor of $0.80 \pm 0.04$ (stat). The control region $\mathrm{CR}_{W 3}$ is obtained by adding the VBF topology selection.

From MC simulation, we expect the fraction of events passing the lepton and jet vetoes and $p_{T}^{\text {miss }}$ selection that also satisfies our VBF topology selection to be the same for the $Z+$ jets and $W+$ jets events. To increase the statistical precision, we combine the two samples and obtain a prediction of $0.008 \pm 0.002$ (stat).

The negligible contribution from QCD multijet production is checked using the number of events passing the analysis selection, except the jet veto and $\mid \Delta \phi\left(\vec{p}_{T}^{\text {miss }}\right.$, jet $\left.{ }_{2}\right) \mid$ requirement. Nonmultijet background $(Z / W+$ jets, $t \bar{t}$, diboson) is subtracted, and the number of events is scaled by the efficiency to inefficiency ratios of the jet veto and $\mid \Delta \phi\left(\vec{p}_{T}^{\text {miss }}\right.$, jet $\left._{2}\right) \mid$ requirements. The two ratios are measured in low- $p_{T}^{\text {miss }}$ multijet-enriched data samples. Other smaller background contributions $\left[Z\left(\rightarrow \ell^{+} \ell^{-}\right)+\right.$jets, $t \bar{t}$, diboson] are taken from simulation.

The dominant source of systematic uncertainty in the background estimate for both $Z(\rightarrow \nu \bar{\nu})+$ jets and $W(\rightarrow$ $\ell \nu)+$ jets comes from the event yields found in the control regions. The control sample statistics lead to an uncertainty in the data-to-MC correction factors of $5-6 \%$, and $24 \%$ on the fraction of events passing the VBF topology selection. Additional sources of systematic uncertainties due to trigger efficiency (5\%), background in the control regions (4-5\%), jet energy resolution and scale (3\%), and integrated luminosity measurement (3\%) [41] are incorporated. The dominant source of systematic uncertainty in the signal expectation comes from the modeling of the two jets in simulation, i.e., the fraction of events passing the VBF topology selection. We take the largest value of the

TABLE I. Event yields predicted from MC in the control regions and observed in the signal region (SR). Only statistical uncertainties are shown. Dashes indicate cases where a background contribution is negligible. The units for the yields are given in the header row of the table.

\begin{tabular}{lccccccc}
\hline \hline Sample & $\mathrm{CR}_{Z 1}\left(10^{3}\right)$ & $\mathrm{CR}_{Z 2}$ & $\mathrm{CR}_{Z 3}$ & $\mathrm{CR}_{W 1}\left(10^{4}\right)$ & $\mathrm{CR}_{W 2}\left(10^{2}\right)$ & $\mathrm{CR}_{W 3}$ & $\mathrm{SR}$ \\
\hline$W(\rightarrow \ell \nu)+$ jets & $0.10 \pm 0.02$ & $0.0_{-0.0}^{+2.4}$ & $0.0_{-0.0}^{+2.4}$ & $6647 \pm 4$ & $13.4 \pm 0.6$ & $8.0 \pm 4.4$ & $43.6 \pm 10.3$ \\
$Z(\rightarrow \nu \bar{\nu})+$ jets & $\cdots$ & $\cdots$ & $\ldots$ & $\ldots$ & $\ldots$ & $\ldots .9$ & $88.2 \pm 9.8$ \\
$Z\left(\rightarrow \ell^{+} \ell^{-}\right)+$jets & $5130 \pm 5$ & $675 \pm 35$ & $5.5 \pm 2.3$ & $594.9 \pm 0.4$ & $0.12 \pm 0.04$ & $0.0_{-0.0}^{+1.9}$ & $0.0_{-0.0}^{+0.2}$ \\
$t \bar{t}$ & $17.2 \pm 0.2$ & $1.3 \pm 1.2$ & $0.0_{-0.0}^{+0.7}$ & $40.5 \pm 0.1$ & $0.13 \pm 0.04$ & $0.0_{-0.0}^{+0.7}$ & $0.0_{-0.0}^{+0.7}$ \\
Diboson & $12.8 \pm 0.1$ & $23.8 \pm 4.9$ & $0.02_{-0.02}^{+0.25}$ & $10.33 \pm 0.03$ & $0.22 \pm 0.01$ & $0.07_{-0.07}^{+0.34}$ & $0.4_{-0.4}^{+0.7}$ \\
$\Sigma$ MC & $5160 \pm 5$ & $700 \pm 36$ & $5.5 \pm 2.3$ & $7292 \pm 5$ & $13.8 \pm 0.6$ & $8.0 \pm 4.4$ & $132 \pm 14$ \\
Data & 5073 & 666 & 6 & 7075 & 11.1 & 9 & 118 \\
\hline \hline
\end{tabular}


observed difference between data and $\mathrm{MC}$ of this fraction from the $Z\left(\rightarrow \mu^{+} \mu^{-}\right)+$jets and $W(\rightarrow \mu \nu)+$ jets control regions, and their uncertainties as an estimate of the signal uncertainty. For the uncertainty due to the choice of parton momentum distributions, we follow the PDF4LHC recommendations [42,43], using CTEQ6.6L, MRST2006, and NNPDF10 [44-46]. The dominant uncertainties that contribute to the signal dijet mass shape include the $p_{T}^{\text {miss }}$ and jet energy scale uncertainties. The background dijet mass shape uncertainties, which vary between $7 \%$ and $42 \%$, are determined by comparing the differences in the predicted and measured dijet mass distributions in various low- $p_{T}^{\text {miss }}$ control regions for $Z$ and $W+$ jets events.

Figure 2 shows the dijet mass distribution after the analysis selection for the backgrounds and the two signal models. Because of the harder scattering required for DM and bottom squark pair production, we expect a harder dijet mass spectrum than for the SM backgrounds. We fit the dijet mass distribution to calculate upper limits on the cross sections at a 95\% confidence level (C.L.), using the $\mathrm{CL}_{s}$ criterion $[47,48]$, with the one-sided (LHC-style) profile likelihood ratio as the test statistic. Systematic uncertainties are represented by nuisance parameters, assuming a gamma or log-normal prior probability for normalization parameters and Gaussian priors for dijet mass shape uncertainties.

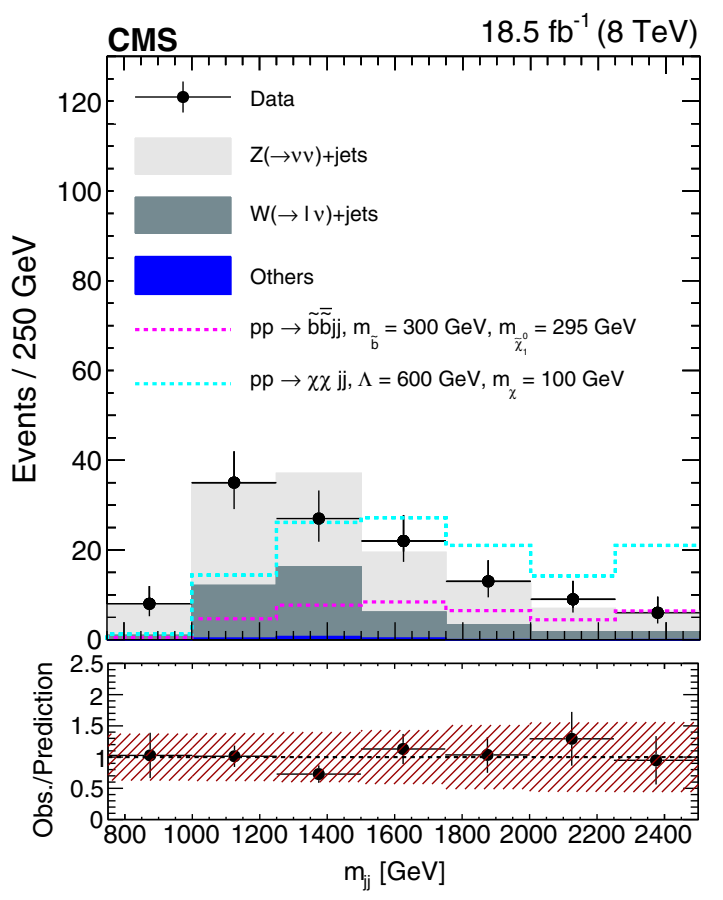

FIG. 2. Dijet mass distribution of the data (dots), estimated background (stacked histograms), and signal samples (dashed lines) after the analysis selection. The last bin includes all events above $2250 \mathrm{GeV}$. The ratio plot (below) shows the yields in data divided by predicted yields for each bin. The shaded band in the ratio plot includes systematic and statistical uncertainties in the background prediction.
The result of the fit for the 95\% C.L. limit in the DM effective theory is given by the solid blue line in Fig. 3 (left); values of $\left(m_{\chi}, \Lambda\right)$ below the curve are excluded. Although EFT is a good approximation in the regime of small momentum transfers, such as direct DM detection experiments, its validity needs to be quantified for LHC experiments where interactions may occur with large momentum transfer. For this purpose, an event in the MC signal sample is classified as having large momentum transfer if the centerof-mass energy of the DM pair $\left(E_{\mathrm{cm}}^{\chi \chi}\right)$ is larger than the mediator mass parameter of the EFT, $\mathcal{M}=\Lambda g_{\mathrm{eff}}$. In the EFT approach, each parameter point of $m_{\gamma}$ and $\Lambda$ is classified as valid if the fraction of MC signal events $\left(R_{\Lambda}\right)$ classified as not having large momentum transfer is $80 \%$ or more. Truncated limits are calculated by adding the requirement $E_{\mathrm{cm}}^{\chi \chi}<\Lambda g_{\mathrm{eff}}$ to the signal acceptance, following Refs. [49,50]. More signal events are removed in higher DM mass regions where $R_{\Lambda}$ curves tend to go up and truncated limits go down. Figure 3 (left) shows curves corresponding to $R_{\Lambda}=80 \%$ and truncated limits for different values of $g_{\text {eff }}$, along with the DM relic abundance $\Omega h^{2}=0.12$, calculated using the MADDM program [51], assuming that DM pairs annihilate to electroweak boson pairs. The DM is more abundant than observed in the regions above or left from the $\Omega h^{2}=0.12$ line.

The observed cross section upper limit on bottom squark pair production in association with two partons $\left(p_{T}>30 \mathrm{GeV},|\Delta \eta|>4.2\right)$ is shown as a function of $m_{\tilde{b}}$ and its difference from $m_{\mathrm{LSP}}$ in Fig. 3 (right). The contours show observed and expected limits on the masses. The excluded mass values are taken at the intersection of the observed cross section limit, with the theoretical cross section less one standard deviation of its uncertainty.

In summary, we have searched for new physics that results in large $p_{T}^{\text {miss }}$ and jets with a VBF topology. The data sample used corresponds to an integrated luminosity of $18.5 \mathrm{fb}^{-1}$, collected by the CMS detector in proton-proton collisions at $\sqrt{s}=8 \mathrm{TeV}$. The low multijet background demonstrates the power of the VBF topology approach for DM and compressed mass spectrum SUSY searches. This is the first search for DM production through pure electroweak VBF processes at a hadron collider. The production of DM via VBF, with masses below $420 \mathrm{GeV}$, is excluded at a $95 \%$ confidence level for a chosen contact interaction scale $\Lambda=600 \mathrm{GeV}$, extending the reach by other DM searches probing similar operators (e.g., $\Lambda$ exclusions up to $\approx 100 \mathrm{GeV}$ for similar DM mass in [8-11]). Limits for different values of $\Lambda$ can be obtained by scaling the $\chi \chi \mathrm{jj}$ cross section, which is proportional to $1 / \Lambda^{2}$. For a nearly mass-degenerate bottom squark and LSP, this analysis sets the most stringent limits reported to date, excluding scalar bottom quarks up to masses of $315 \mathrm{GeV}$ at a $95 \%$ confidence level. 

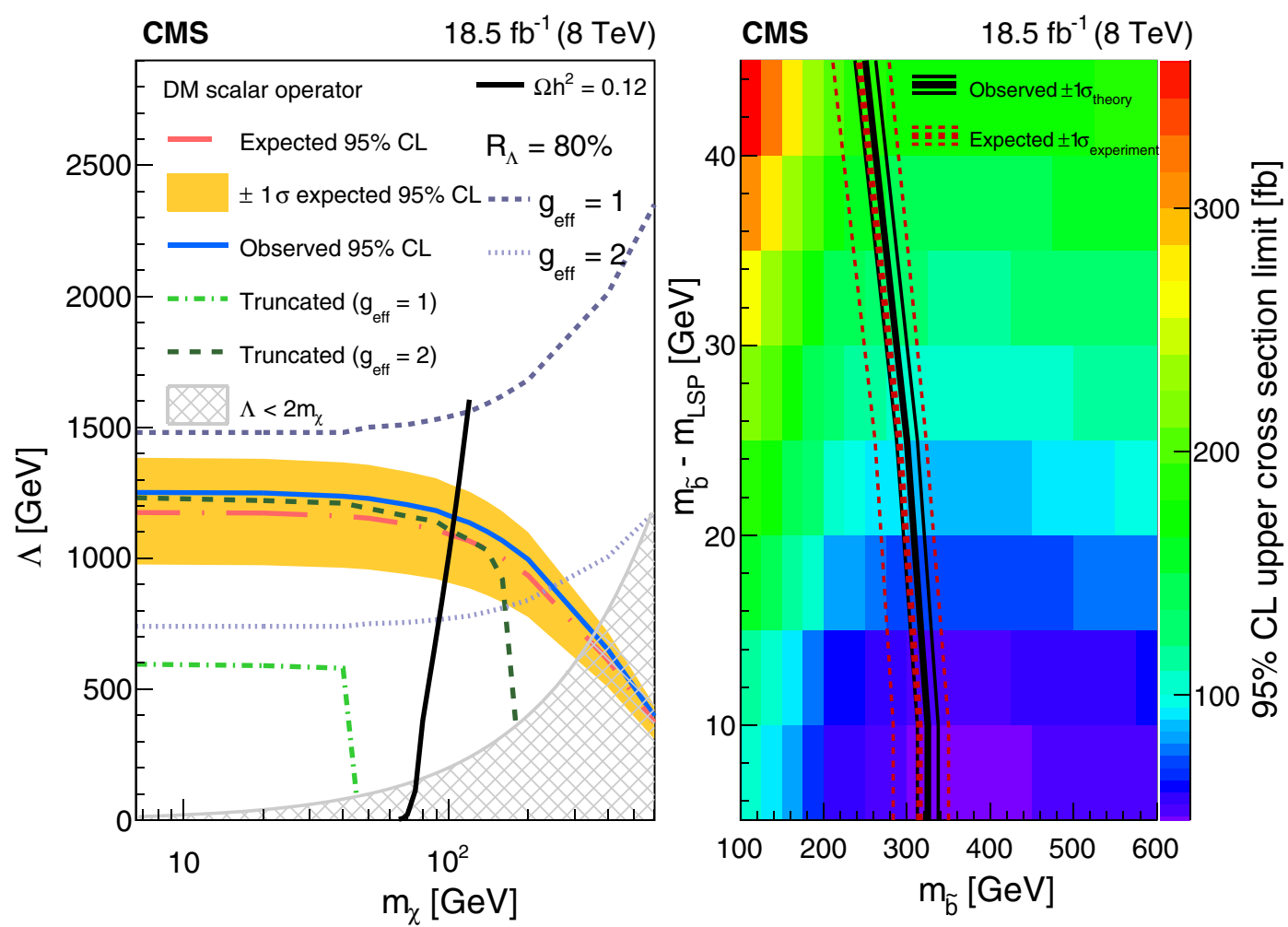

FIG. 3. (left) Contact interaction scale limit at $95 \%$ C.L. as a function of the DM mass. The validity of the effective field theory is quantified by (i) $R_{\Lambda}=80 \%$ contours and (ii) truncated limits for different values of the effective coupling. The DM relic abundance $\Omega h^{2}=0.12$ is calculated as described in the text. (right) Bottom squark pair production 95\% C.L. upper cross section limit as a function of the bottom squark mass and the mass difference between the bottom squark and the LSP. The observed (expected) cross section limit includes one standard deviation bands for the theoretical (experimental) uncertainty.

We congratulate our colleagues in the CERN accelerator departments for the excellent performance of the LHC and thank the technical and administrative staffs at CERN and at other CMS institutes for their contributions to the success of the CMS effort. In addition, we gratefully acknowledge the computing centers and personnel of the Worldwide LHC Computing Grid for delivering so effectively the computing infrastructure essential to our analyses. Finally, we acknowledge the enduring support for the construction and operation of the LHC and the CMS detector provided by the following funding agencies: BMWFW and FWF (Austria); FNRS and FWO (Belgium); CNPq, CAPES, FAPERJ, and FAPESP (Brazil); MES (Bulgaria); CERN; CAS, MoST, and NSFC (China); COLCIENCIAS (Colombia); MSES and Croatian Science Foundation CSF (Croatia); RPF (Cyprus); MoER, ERC IUT and ERDF (Estonia); Academy of Finland, MEC, and HIP (Finland); CEA and CNRS/IN2P3 (France); BMBF, DFG, and HGF (Germany); GSRT (Greece); OTKA and NIH (Hungary); DAE and DST (India); IPM (Iran); SFI (Ireland); INFN (Italy); MSIP and NRF (Republic of Korea); LAS (Lithuania); MOE and UM (Malaysia); BUAP, CINVESTAV, CONACYT, LNS, SEP, and UASLP-FAI (Mexico); MBIE (New Zealand); PAEC (Pakistan); MSHE and NSC (Poland); FCT (Portugal);
JINR (Dubna); MON, RosAtom, RAS and RFBR (Russia); MESTD (Serbia); SEIDI and CPAN (Spain); Swiss Funding Agencies (Switzerland); MST (Taipei); ThEPCenter, IPST, STAR and NSTDA (Thailand); TUBITAK and TAEK (Turkey); NASU and SFFR (Ukraine); STFC (United Kingdom); DOE and NSF (USA). Individuals have received support from the Marie-Curie program and the European Research Council and EPLANET (European Union); the Leventis Foundation; the A. P. Sloan Foundation; the Alexander von Humboldt Foundation; the Belgian Federal Science Policy Office; the Fonds pour la Formation à la Recherche dans l'Industrie et dans l'Agriculture (FRIA-Belgium); the Agentschap voor Innovatie door Wetenschap en Technologie (IWT-Belgium); the Ministry of Education, Youth and Sports (MEYS) of the Czech Republic; the Council of Science and Industrial Research, India; the HOMING PLUS program of the Foundation for Polish Science, cofinanced from European Union, Regional Development Fund; the Mobility Plus program of the Ministry of Science and Higher Education (Poland); the OPUS program of the National Science Center (Poland); the Thalis and Aristeia programs cofinanced by EU-ESF and the Greek NSRF; the National Priorities Research Program by Qatar National Research Fund; the Programa 
Clarín-COFUND del Principado de Asturias; the Rachadapisek Sompot Fund for Postdoctoral Fellowship, Chulalongkorn University (Thailand); the Chulalongkorn Academic into Its 2nd Century Project Advancement Project (Thailand); and the Welch Foundation, Contract No. C-1845.

[1] P. A. R. Ade et al., Planck 2013 results. XVI. cosmological parameters, Astron. Astrophys. 571, A16 (2014).

[2] S. P. Martin, A supersymmetry primer, Adv. Ser. Dir. High Energy Phys. 18, 1 (1998).

[3] G. R. Farrar and P. Fayet, Phenomenology of the production, decay, and detection of new hadronic states associated with supersymmetry, Phys. Lett. B 76, 575 (1978).

[4] CMS Collaboration, Searches for electroweak production of charginos, neutralinos, and sleptons decaying to leptons and $\mathrm{W}, \mathrm{Z}$, and Higgs bosons in $p p$ collisions at $8 \mathrm{TeV}$, Eur. Phys. J. C 74, 3036 (2014).

[5] CMS Collaboration, Search for supersymmetry in events with soft leptons, low jet multiplicity, and missing transverse momentum in proton-proton collisions at $\sqrt{s}=8 \mathrm{TeV}$, Phys. Lett. B 759, 9 (2016).

[6] ATLAS Collaboration, Search for direct production of charginos and neutralinos in events with three leptons and missing transverse momentum in $\sqrt{s}=8 \mathrm{TeV} p p$ collisions with the ATLAS detector, J. High Energy Phys. 04 (2014) 169.

[7] K. A. Olive et al. (Particle Data Group), Review of particle physics, Chin. Phys. C 38, 090001 (2014).

[8] ATLAS Collaboration, Search for Dark Matter in Events with Missing Transverse Momentum and a Higgs Boson Decaying to Two Photons in $p p$ Collisions at $\sqrt{s}=8 \mathrm{TeV}$ with the Atlas Detector, Phys. Rev. Lett. 115, 131801 (2015).

[9] ATLAS Collaboration, Search for dark matter produced in association with a Higgs boson decaying to two bottom quarks in $p p$ collisions at $\sqrt{s}=8 \mathrm{TeV}$ with the atlas detector, Phys. Rev. D 93, 072007 (2016).

[10] ATLAS Collaboration, Search for Dark Matter in Events with a Hadronically Decaying $\mathrm{W}$ or $\mathrm{Z}$ Boson and Missing Transverse Momentum in $p p$ Collisions at $\sqrt{s}=8 \mathrm{TeV}$ with the Atlas Detector, Phys. Rev. Lett. 112, 041802 (2014).

[11] ATLAS Collaboration, Search for dark matter in events with a $\mathrm{z}$ boson and missing transverse momentum in $p p$ collisions at $\sqrt{s}=8 \mathrm{TeV}$ with the atlas detector, Phys. Rev. D 90, 012004 (2014).

[12] R. C. Cotta, J. L. Hewett, M. P. Le, and T. G. Rizzo, Bounds on dark matter interactions with electroweak gauge bosons, Phys. Rev. D 88, 116009 (2013).

[13] CMS Collaboration, Searches for third-generation squark production in fully hadronic final states in proton-proton collisions at $\sqrt{s}=8 \mathrm{TeV}$, J. High Energy Phys. 06 (2015) 116.

[14] ATLAS Collaboration, Search for new phenomena in final states with an energetic jet and large missing transverse momentum in $p p$ collisions at $\sqrt{s}=8 \mathrm{TeV}$ with the ATLAS detector, Eur. Phys. J. C 75, 299 (2015).
[15] CMS Collaboration, Search for dark matter, extra dimensions, and unparticles in monojet events in proton-proton collisions at $\sqrt{s}=8 \mathrm{TeV}$, Eur. Phys. J. C 75, 235 (2015).

[16] B. Dutta, A. Gurrola, W. Johns, T. Kamon, P. Sheldon, and K. Sinha, Vector boson fusion processes as a probe of supersymmetric electroweak sectors at the LHC, Phys. Rev. D 87, 035029 (2013).

[17] A. G. Delannoy, B. Dutta, A. Gurrola, W. Johns, T. Kamon, E. Luiggi, A. Melo, P. Sheldon, K. Sinha, K. Wang, and S. $\mathrm{Wu}$, Probing Dark Matter at the LHC using Vector Boson Fusion Processes, Phys. Rev. Lett. 111, 061801 (2013).

[18] CMS Collaboration, Search for supersymmetry in the vector-boson fusion topology in proton-proton collisions at $\sqrt{s}=8 \mathrm{TeV}$, J. High Energy Phys. 11 (2015) 189.

[19] ATLAS Collaboration, Search for the electroweak production of supersymmetric particles in $\sqrt{s}=8 \mathrm{TeV} p p$ collisions with the ATLAS detector, Phys. Rev. D 93, 052002 (2016).

[20] CMS Collaboration, Search for invisible decays of Higgs bosons in the vector boson fusion and associated $\mathrm{ZH}$ production modes, Eur. Phys. J. C 74, 2980 (2014).

[21] ATLAS Collaboration, Search for invisible decays of a Higgs boson using vector-boson fusion in $p p$ collisions at $\sqrt{s}=8 \mathrm{TeV}$ with the ATLAS detector, J. High Energy Phys. 01 (2016) 172.

[22] CMS Collaboration, The CMS experiment at the CERN LHC, J. Instrum. 3, S08004 (2008).

[23] CMS Collaboration, CMS Physics Analysis Summary Report No. CMS-PAS-PFT-09-001, 2009, http://cdsweb .cern.ch/record/1194487.

[24] CMS Collaboration, CMS Physics Analysis Summary Report No. CMS-PAS-PFT-10-001, 2010, http://cdsweb .cern.ch/record/1247373.

[25] M. Cacciari, G. P. Salam, and G. Soyez, The anti- $k_{t}$ jet clustering algorithm, J. High Energy Phys. 04 (2008) 063.

[26] CMS Collaboration, CMS Physics Analysis Summary Report No. CMS-PAS-JME-13-005, 2013, http://cds.cern .ch/record/1581583.

[27] CMS Collaboration, Identification of b-quark jets with the CMS experiment, J. Instrum. 8, P04013 (2013).

[28] CMS Collaboration, CMS Physics Analysis Summary Report No. CMS-PAS-BTV-13-001, 2013, http://cds.cern .ch/record/1581306.

[29] CMS Collaboration, Performance of electron reconstruction and selection with the CMS detector in proton-proton collisions at $\sqrt{s}=8 \mathrm{TeV}$, J. Instrum. 10, P06005 (2015).

[30] CMS Collaboration, Performance of CMS muon reconstruction in $p p$ collision events at $\sqrt{s}=7 \mathrm{TeV}$, J. Instrum. 7, P10002 (2012).

[31] CMS Collaboration, Performance of tau-lepton reconstruction and identification in CMS, J. Instrum. 7, P01001 (2012).

[32] J. Alwall, R. Frederix, S. Frixione, V. Hirschi, F. Maltoni, O. Mattelaer, H. S. Shao, T. Stelzer, P. Torrielli, and M. Zaro, The automated computation of tree-level and next-to-leading order differential cross sections, and their matching to parton shower simulations, J. High Energy Phys. 07 (2014) 079.

[33] P. Nason and C. Oleari, NLO Higgs boson production via vector-boson fusion matched with shower in POWHEG, J. High Energy Phys. 02 (2010) 037. 
[34] S. Alioli, P. Nason, C. Oleari, and E. Re, A general framework for implementing NLO calculations in shower Monte Carlo programs: the POWHEG BOX, J. High Energy Phys. 06 (2010) 043.

[35] J. Pumplin, D. R. Stump, J. Huston, H. L. Lai, Pavel M. Nadolsky, and W. K. Tung, New generation of parton distributions with uncertainties from global QCD analysis, J. High Energy Phys. 07 (2002) 012.

[36] H.-L. Lai, M. Guzzi, J. Huston, Z. Li, P. M. Nadolsky, J. Pumplin, and C.P. Yuan, New parton distributions for collider physics, Phys. Rev. D 82, 074024 (2010).

[37] T. Sjöstrand, S. Mrenna, and P.Z. Skands, PYTHIA 6.4 physics and manual, J. High Energy Phys. 05 (2006) 026.

[38] S. Agostinelli et al., GEANT4-A simulation toolkit, Nucl. Instrum. Methods Phys. Res., Sect. A 506, 250 (2003).

[39] CMS Collaboration in Proceedings, 18th International Conference on Computing in High Energy and Nuclear Physics CHEP, 2010; S. Abdullin, P. Azzi, F. Beaudette, P. Janot, and A. Perrotta (on behalf of the CMS Collaboration), The Fast Simulation of the CMS Detector at LHC, J. Phys. Conf. Ser. 331, 032049 (2011)].

[40] W. Beenakker, M. Krämer, T. Plehn, M. Spira, and P. M. Zerwas, Stop production at hadron colliders, Nucl. Phys. B515, 3 (1998).

[41] CMS Collaboration, CMS Physics Analysis Summary Report No. CMS-PAS-LUM-13-001, 2013, http://cdsweb .cern.ch/record/1598864.

[42] S. Alekhin et al., The PDF4LHC Working Group Interim Report, arXiv:1101.0536.
[43] M. Botje et al., The PDF4LHC Working Group Interim Recommendations, arXiv:1101.0538.

[44] P. M. Nadolsky, H.-L. Lai, Q.-H. Cao, J. Huston, J. Pumplin, D. Stump, W.-K. Tung, and C. P. Yuan, Implications of CTEQ global analysis for collider observables, Phys. Rev. D 78, 013004 (2008).

[45] A. D. Martin, W. J. Stirling, R. S. Thorne, and G. Watt, Update of parton distributions at NNLO, Phys. Lett. B 652, 292 (2007).

[46] R. D. Ball, L. Del Debbio, S. Forte, A. Guffanti, J. Latorre, A. Piccione, J. Rojo, and M. Ubiali, A determination of parton distributions with faithful uncertainty estimation, Nucl. Phys. B809, 1 (2009).

[47] A. L. Read, Presentation of search results: The $C L_{s}$ technique, J. Phys. G 28, 2693 (2002).

[48] T. Junk, Confidence level computation for combining searches with small statistics, Nucl. Instrum. Methods Phys. Res., Sect. A 434, 435 (1999).

[49] Daniel Abercrombie et al., Dark Matter Benchmark Models for Early LHC Run-2 Searches: Report of the ATLAS/CMS Dark Matter Forum, arXiv:1507.00966.

[50] CMS Collaboration, Search for dark matter and unparticles produced in association with a $Z$ boson in proton-proton collisions at $\sqrt{s}=8 \mathrm{TeV}$, Phys. Rev. D 93, 052011 (2016).

[51] M. Backović, K. Kong, and M. McCaskey, MADDM v.1.0: Computation of dark matter relic abundance using MadGraph 5, Phys. Dark Univ. 5-6, 18 (2014).

V. Khachatryan, ${ }^{1}$ A. M. Sirunyan, ${ }^{1}$ A. Tumasyan, ${ }^{1}$ W. Adam, ${ }^{2}$ E. Asilar, ${ }^{2}$ T. Bergauer, ${ }^{2}$ J. Brandstetter, ${ }^{2}$ E. Brondolin, ${ }^{2}$ M. Dragicevic, ${ }^{2}$ J. Erö, ${ }^{2}$ M. Flechl, ${ }^{2}$ M. Friedl, ${ }^{2}$ R. Frühwirth, ${ }^{2, b}$ V. M. Ghete, ${ }^{2}$ C. Hartl, ${ }^{2}$ N. Hörmann, ${ }^{2}$ J. Hrubec, ${ }^{2}$ M. Jeitler, ${ }^{2, b}$ A. König, ${ }^{2}$ I. Krätschmer, ${ }^{2}$ D. Liko, ${ }^{2}$ T. Matsushita, ${ }^{2}$ I. Mikulec, ${ }^{2}$ D. Rabady, ${ }^{2}$ N. Rad, ${ }^{2}$ B. Rahbaran, ${ }^{2}$ H. Rohringer, ${ }^{2}$ J. Schieck, ${ }^{2, b}$ J. Strauss, ${ }^{2}$ W. Treberer-Treberspurg, ${ }^{2}$ W. Waltenberger, ${ }^{2}$ C.-E. Wulz, ${ }^{2, b}$ V. Mossolov, ${ }^{3}$ N. Shumeiko, ${ }^{3}$ J. Suarez Gonzalez, ${ }^{3}$ S. Alderweireldt, ${ }^{4}$ T. Cornelis, ${ }^{4}$ E. A. De Wolf, ${ }^{4}$ X. Janssen, ${ }^{4}$ A. Knutsson, ${ }^{4}$ J. Lauwers, ${ }^{4}$ S. Luyckx, ${ }^{4}$ M. Van De Klundert, ${ }^{4}$ H. Van Haevermaet, ${ }^{4}$ P. Van Mechelen, ${ }^{4}$ N. Van Remortel, ${ }^{4}$ A. Van Spilbeeck, ${ }^{4}$ S. Abu Zeid, ${ }^{5}$ F. Blekman, ${ }^{5}$ J. D'Hondt, ${ }^{5}$ N. Daci, ${ }^{5}$ I. De Bruyn, ${ }^{5}$ K. Deroover, ${ }^{5}$ N. Heracleous, ${ }^{5}$ S. Lowette, ${ }^{5}$ S. Moortgat, ${ }^{5}$ L. Moreels, ${ }^{5}$ A. Olbrechts, ${ }^{5}$ Q. Python, ${ }^{5}$ S. Tavernier, ${ }^{5}$ W. Van Doninck, ${ }^{5}$ P. Van Mulders, ${ }^{5}$ I. Van Parijs, ${ }^{5}$ H. Brun,${ }^{6}$ C. Caillol, ${ }^{6}$ B. Clerbaux,${ }^{6}$ G. De Lentdecker, ${ }^{6}$ H. Delannoy, ${ }^{6}$ G. Fasanella, ${ }^{6}$ L. Favart, ${ }^{6}$ R. Goldouzian, ${ }^{6}$ A. Grebenyuk, ${ }^{6}$ G. Karapostoli, ${ }^{6}$ T. Lenzi, ${ }^{6}$ A. Léonard, ${ }^{6}$ T. Maerschalk, ${ }^{6}$ A. Marinov, ${ }^{6}$ A. Randle-conde, ${ }^{6}$ T. Seva ${ }^{6}$ C. Vander Velde, ${ }^{6}$ P. Vanlaer, ${ }^{6}$ R. Yonamine, ${ }^{6}$ F. Zenoni ${ }^{6}{ }^{6}$. Zhang, ${ }^{6, c}$ A. Cimmino, ${ }^{7}$ D. Dobur, ${ }^{7}$ A. Fagot, ${ }^{7}$ G. Garcia, ${ }^{7}$ M. Gul, ${ }^{7}$ J. Mccartin, ${ }^{7}$ D. Poyraz, ${ }^{7}$ S. Salva, ${ }^{7}$ R. Schöfbeck, ${ }^{7}$ M. Tytgat, ${ }^{7}$ W. Van Driessche, ${ }^{7}$ E. Yazgan, ${ }^{7}$ N. Zaganidis, ${ }^{7}$ C. Beluffi, ${ }^{8, d}$ O. Bondu, ${ }^{8}$ S. Brochet, ${ }^{8}$ G. Bruno, ${ }^{8}$ A. Caudron, ${ }^{8}$ L. Ceard ${ }^{8}$ S. De Visscher, ${ }^{8}$ C. Delaere, ${ }^{8}$ M. Delcourt, ${ }^{8}$ L. Forthomme ${ }^{8}$ B. Francois, ${ }^{8}$ A. Giammanco, ${ }^{8}$ A. Jafari, ${ }^{8}$ P. Jez,${ }^{8}$ M. Komm, ${ }^{8}$ V. Lemaitre,${ }^{8}$ A. Magitteri, ${ }^{8}$ A. Mertens, ${ }^{8}$ M. Musich, ${ }^{8}$ C. Nuttens, ${ }^{8}$ K. Piotrzkowski, ${ }^{8}$ L. Quertenmont,${ }^{8}$ M. Selvaggi, ${ }^{8}$ M. Vidal Marono, ${ }^{8}$ S. Wertz, ${ }^{8}$ N. Beliy, ${ }^{9}$ W. L. Aldá Júnior, ${ }^{10}$ F. L. Alves, ${ }^{10}$ G. A. Alves, ${ }^{10}$ L. Brito, ${ }^{10}$ M. Correa Martins Junior, ${ }^{10}$ C. Hensel, ${ }^{10}$ A. Moraes, ${ }^{10}$ M. E. Pol,,${ }^{10}$ P. Rebello Teles, ${ }^{10}$ E. Belchior Batista Das Chagas, ${ }^{11}$ W. Carvalho, ${ }^{11}$ J. Chinellato, ${ }^{11, e}$ A. Custódio, ${ }^{11}$ E. M. Da Costa, ${ }^{11}$ G. G. Da Silveira, ${ }^{11}$ D. De Jesus Damiao, ${ }^{11}$ C. De Oliveira Martins, ${ }^{11}$ S. Fonseca De Souza, ${ }^{11}$ L. M. Huertas Guativa, ${ }^{11}$ H. Malbouisson, ${ }^{11}$ D. Matos Figueiredo, ${ }^{11}$ C. Mora Herrera, ${ }^{11}$ L. Mundim, ${ }^{11}$ H. Nogima, ${ }^{11}$ W. L. Prado Da Silva, ${ }^{11}$ A. Santoro, ${ }^{11}$ A. Sznajder, ${ }^{11}$ E. J. Tonelli Manganote,,${ }^{11, e}$ A. Vilela Pereira, ${ }^{11}$ S. Ahuja, ${ }^{12 a}$ C. A. Bernardes, ${ }^{12 b}$ S. Dogra, ${ }^{12 a}$ T. R. Fernandez Perez Tomei, ${ }^{12 a}$ E. M. Gregores, ${ }^{12 b}$ P. G. Mercadante, ${ }^{12 b}$ C. S. Moon, ${ }^{12 a, f}$ S. F. Novaes, ${ }^{12 a}$ Sandra S. Padula, ${ }^{12 a}$ D. Romero Abad, ${ }^{12 b}$ J. C. Ruiz Vargas, ${ }^{12 a}$ 
A. Aleksandrov,${ }^{13}$ R. Hadjiiska, ${ }^{13}$ P. Iaydjiev, ${ }^{13}$ M. Rodozov, ${ }^{13}$ S. Stoykova, ${ }^{13}$ G. Sultanov,${ }^{13}$ M. Vutova, ${ }^{13}$ A. Dimitrov,${ }^{14}$ I. Glushkov, ${ }^{14}$ L. Litov, ${ }^{14}$ B. Pavlov, ${ }^{14}$ P. Petkov, ${ }^{14}$ W. Fang, ${ }^{15, g}$ M. Ahmad,${ }^{16}$ J. G. Bian, ${ }^{16}$ G. M. Chen, ${ }^{16}$ H. S. Chen, ${ }^{16}$ M. Chen, ${ }^{16}$ Y. Chen, ${ }^{16,}$ T. Cheng, ${ }^{16}$ R. Du, ${ }^{16}$ C. H. Jiang, ${ }^{16}$ D. Leggat,${ }^{16}$ Z. Liu,,${ }^{16}$ F. Romeo, ${ }^{16}$ S. M. Shaheen, ${ }^{16}$ A. Spiezia, ${ }^{16}$ J. Tao, ${ }^{16}$ C. Wang, ${ }^{16}$ Z. Wang, ${ }^{16}$ H. Zhang, ${ }^{16}$ J. Zhao, ${ }^{16}$ C. Asawatangtrakuldee,${ }^{17}$ Y. Ban, ${ }^{17}$ Q. Li,${ }^{17}$ S. Liu, ${ }^{17}$ Y. Mao, ${ }^{17}$ S. J. Qian, ${ }^{17}$ D. Wang, ${ }^{17}$ Z. Xu, ${ }^{17}$ C. Avila, ${ }^{18}$ A. Cabrera, ${ }^{18}$ L. F. Chaparro Sierra, ${ }^{18}$ C. Florez, ${ }^{18}$ J. P. Gomez, ${ }^{18}$

J. D. Ruiz Alvarez, ${ }^{18}$ J. C. Sanabria, ${ }^{18}$ N. Godinovic, ${ }^{19}$ D. Lelas,${ }^{19}$ I. Puljak,${ }^{19}$ P. M. Ribeiro Cipriano, ${ }^{19}$ Z. Antunovic,${ }^{20}$ M. Kovac, ${ }^{20}$ V. Brigljevic, ${ }^{21}$ D. Ferencek, ${ }^{21}$ K. Kadija, ${ }^{21}$ J. Luetic, ${ }^{21}$ S. Micanovic, ${ }^{21}$ L. Sudic, ${ }^{21}$ A. Attikis, ${ }^{22}$ G. Mavromanolakis, ${ }^{22}$ J. Mousa, ${ }^{22}$ C. Nicolaou, ${ }^{22}$ F. Ptochos, ${ }^{22}$ P. A. Razis, ${ }^{22}$ H. Rykaczewski, ${ }^{22}$ M. Finger, ${ }^{23, i}$ M. Finger Jr., ${ }^{23, i}$ E. Carrera Jarrin, ${ }^{24}$ A. Awad, ${ }^{25}$ S. Elgammal, ${ }^{25, j}$ A. Mohamed, ${ }^{25, k}$ E. Salama, ${ }^{25, j, 1}$ B. Calpas, ${ }^{26}$ M. Kadastik, ${ }^{26}$ M. Murumaa, ${ }^{26}$ L. Perrini, ${ }^{26}$ M. Raidal, ${ }^{26}$ A. Tiko, ${ }^{26}$ C. Veelken, ${ }^{26}$ P. Eerola, ${ }^{27}$ J. Pekkanen, ${ }^{27}$ M. Voutilainen, ${ }^{27}$ J. Härkönen, ${ }^{28}$ V. Karimäki ${ }^{28}$ R. Kinnunen, ${ }^{28}$ T. Lampén, ${ }^{28}$ K. Lassila-Perini, ${ }^{28}$ S. Lehti, ${ }^{28}$ T. Lindén, ${ }^{28}$ P. Luukka, ${ }^{28}$ T. Peltola, ${ }^{28}$ J. Tuominiemi, ${ }^{28}$ E. Tuovinen, ${ }^{28}$ L. Wendland ${ }^{28}$ J. Talvitie, ${ }^{29}$ T. Tuuva, ${ }^{29}$ M. Besancon, ${ }^{30}$ F. Couderc ${ }^{30}$ M. Dejardin ${ }^{30}$ D. Denegri, ${ }^{30}$ B. Fabbro, ${ }^{30}$ J. L. Faure, ${ }^{30}$ C. Favaro,${ }^{30}$ F. Ferri, ${ }^{30}$ S. Ganjour,${ }^{30}$ S. Ghosh ${ }^{30}$ A. Givernaud, ${ }^{30}$ P. Gras, ${ }^{30}$ G. Hamel de Monchenault, ${ }^{30}$ P. Jarry, ${ }^{30}$ E. Locci, ${ }^{30}$ M. Machet, ${ }^{30}$ J. Malcles,${ }^{30}$ J. Rander, ${ }^{30}$ A. Rosowsky, ${ }^{30}$ M. Titov, ${ }^{30}$ A. Zghiche, ${ }^{30}$ A. Abdulsalam, ${ }^{31}$ I. Antropov, ${ }^{31}$ S. Baffioni, ${ }^{31}$ F. Beaudette,${ }^{31}$ P. Busson, ${ }^{31}$ L. Cadamuro, ${ }^{31}$ E. Chapon, ${ }^{31}$ C. Charlot, ${ }^{31}$ O. Davignon, ${ }^{31}$ R. Granier de Cassagnac, ${ }^{31}$ M. Jo, ${ }^{31}$ S. Lisniak, ${ }^{31}$ P. Miné,${ }^{31}$ I. N. Naranjo, ${ }^{31}$ M. Nguyen, ${ }^{31}$ C. Ochando,${ }^{31}$ G. Ortona, ${ }^{31}$ P. Paganini, ${ }^{31}$ P. Pigard,${ }^{31}$ S. Regnard,${ }^{31}$ R. Salerno,${ }^{31}$ Y. Sirois, ${ }^{31}$ T. Strebler, ${ }^{31}$ Y. Yilmaz, ${ }^{31}$ A. Zabi, ${ }^{31}$ J.-L. Agram, ${ }^{32, m}$ J. Andrea, ${ }^{32}$ A. Aubin, ${ }^{32}$ D. Bloch, ${ }^{32}$ J.-M. Brom, ${ }^{32}$ M. Buttignol, ${ }^{32}$ E. C. Chabert, ${ }^{32}$ N. Chanon, ${ }^{32}$ C. Collard, ${ }^{32}$ E. Conte, ${ }^{32, \mathrm{~m}}$ X. Coubez, ${ }^{32}$ J.-C. Fontaine, ${ }^{32, \mathrm{~m}}$ D. Gelé, ${ }^{32}$ U. Goerlach, ${ }^{32}$ A.-C. Le Bihan, ${ }^{32}$ J. A. Merlin, ${ }^{32, n}$ K. Skovpen, ${ }^{32}$ P. Van Hove, ${ }^{32}$ S. Gadrat, ${ }^{33}$ S. Beauceron, ${ }^{34}$ C. Bernet, ${ }^{34}$ G. Boudoul, ${ }^{34}$ E. Bouvier, ${ }^{34}$ C. A. Carrillo Montoya,${ }^{34}$ R. Chierici, ${ }^{34}$ D. Contardo,${ }^{34}$ B. Courbon, ${ }^{34}$ P. Depasse, ${ }^{34}$ H. El Mamouni, ${ }^{34}$ J. Fan, ${ }^{34}$ J. Fay, ${ }^{34}$ S. Gascon, ${ }^{34}$ M. Gouzevitch, ${ }^{34}$ G. Grenier, ${ }^{34}$ B. Ille, ${ }^{34}$ F. Lagarde, ${ }^{34}$ I. B. Laktineh, ${ }^{34}$ M. Lethuillier, ${ }^{34}$ L. Mirabito, ${ }^{34}$ A. L. Pequegnot, ${ }^{34}$ S. Perries, ${ }^{34}$ A. Popov, ${ }^{34, o}$ D. Sabes,${ }^{34}$ V. Sordini, ${ }^{34}$ M. Vander Donckt, ${ }^{34}$ P. Verdier, ${ }^{34}$ S. Viret, ${ }^{34}$ T. Toriashvili, ${ }^{35, p}$ Z. Tsamalaidze, ${ }^{36, i}$ C. Autermann, ${ }^{37}$ S. Beranek, ${ }^{37}$ L. Feld, ${ }^{37}$ A. Heister, ${ }^{37}$ M. K. Kiesel, ${ }^{37}$ K. Klein, ${ }^{37}$ M. Lipinski, ${ }^{37}$ A. Ostapchuk, ${ }^{37}$ M. Preuten, ${ }^{37}$ F. Raupach, ${ }^{37}$ S. Schael, ${ }^{37}$ C. Schomakers, ${ }^{37}$ J. F. Schulte, ${ }^{37}$ J. Schulz, ${ }^{37}$ T. Verlage, ${ }^{37}$ H. Weber, ${ }^{37}$ V. Zhukov, ${ }^{37,0}$ M. Ata, ${ }^{38}$ M. Brodski, ${ }^{38}$ E. Dietz-Laursonn, ${ }^{38}$ D. Duchardt, ${ }^{38}$ M. Endres, ${ }^{38}$ M. Erdmann, ${ }^{38}$ S. Erdweg, ${ }^{38}$ T. Esch, ${ }^{38}$ R. Fischer, ${ }^{38}$ A. Güth, ${ }^{38}$ T. Hebbeker ${ }^{38}$ C. Heidemann, ${ }^{38}$ K. Hoepfner ${ }^{38}$ S. Knutzen, ${ }^{38}$ M. Merschmeyer, ${ }^{38}$ A. Meyer, ${ }^{38}$ P. Millet, ${ }^{38}$ S. Mukherjee, ${ }^{38}$ M. Olschewski, ${ }^{38}$ K. Padeken,${ }^{38}$ P. Papacz, ${ }^{38}$ T. Pook, ${ }^{38}$ M. Radziej, ${ }^{38}$ H. Reithler, ${ }^{38}$ M. Rieger, ${ }^{38}$ F. Scheuch,${ }^{38}$ L. Sonnenschein, ${ }^{38}$ D. Teyssier, ${ }^{38}$ S. Thüer,${ }^{38}$ V. Cherepanov, ${ }^{39}$ Y. Erdogan, ${ }^{39}$ G. Flügge,${ }^{39}$ H. Geenen, ${ }^{39}$ M. Geisler ${ }^{39}$ F. Hoehle,${ }^{39}$ B. Kargoll,${ }^{39}$ T. Kress, ${ }^{39}$ A. Künsken, ${ }^{39}$ J. Lingemann, ${ }^{39}$ A. Nehrkorn, ${ }^{39}$ A. Nowack, ${ }^{39}$ I. M. Nugent, ${ }^{39}$ C. Pistone, ${ }^{39}$ O. Pooth, ${ }^{39}$ A. Stahl,,${ }^{39, n}$ M. Aldaya Martin, ${ }^{40}$ I. Asin, ${ }^{40}$ K. Beernaert, ${ }^{40}$ O. Behnke, ${ }^{40}$ U. Behrens, ${ }^{40}$ A. A. Bin Anuar, ${ }^{40}$ K. Borras, ${ }^{40, q}$ A. Campbell, ${ }^{40}$ P. Connor, ${ }^{40}$ C. Contreras-Campana, ${ }^{40}$ F. Costanza,${ }^{40}$ C. Diez Pardos,${ }^{40}$ G. Dolinska, ${ }^{40}$ G. Eckerlin, ${ }^{40}$ D. Eckstein, ${ }^{40}$ T. Eichhorn, ${ }^{40}$ E. Gallo, ${ }^{40, r}$ J. Garay Garcia, ${ }^{40}$ A. Geiser, ${ }^{40}$ A. Gizhko, ${ }^{40}$ J. M. Grados Luyando, ${ }^{40}$ P. Gunnellini, ${ }^{40}$ A. Harb, ${ }^{40}$ J. Hauk, ${ }^{40}$ M. Hempel, ${ }^{40, s}$ H. Jung, ${ }^{40}$ A. Kalogeropoulos,${ }^{40}$ O. Karacheban, ${ }^{40, s}$ M. Kasemann, ${ }^{40}$ J. Kieseler, ${ }^{40}$ C. Kleinwort, ${ }^{40}$

I. Korol, ${ }^{40}$ W. Lange, ${ }^{40}$ A. Lelek, ${ }^{40}$ J. Leonard, ${ }^{40}$ K. Lipka, ${ }^{40}$ A. Lobanov,${ }^{40}$ W. Lohmann, ${ }^{40, s}$ R. Mankel, ${ }^{40}$ I.-A. Melzer-Pellmann, ${ }^{40}$ A. B. Meyer, ${ }^{40}$ G. Mittag, ${ }^{40}$ J. Mnich ${ }^{40}$ A. Mussgiller, ${ }^{40}$ E. Ntomari, ${ }^{40}$ D. Pitzl,${ }^{40}$ R. Placakyte ${ }^{40}$ A. Raspereza, ${ }^{40}$ B. Roland, ${ }^{40}$ M. Ö. Sahin,${ }^{40}$ P. Saxena, ${ }^{40}$ T. Schoerner-Sadenius,${ }^{40}$ C. Seitz, ${ }^{40}$ S. Spannagel,,${ }^{40}$ N. Stefaniuk,${ }^{40}$ K. D. Trippkewitz,${ }^{40}$ G. P. Van Onsem, ${ }^{40}$ R. Walsh, ${ }^{40}$ C. Wissing, ${ }^{40}$ V. Blobel,,${ }^{41}$ M. Centis Vignali, ${ }^{41}$ A. R. Draeger, ${ }^{41}$ T. Dreyer, ${ }^{41}$ J. Erfle, ${ }^{41}$ E. Garutti, ${ }^{41}$ K. Goebel,${ }^{41}$ D. Gonzalez,${ }^{41}$ M. Görner, ${ }^{41}$ J. Haller ${ }^{41}$ M. Hoffmann, ${ }^{41}$ R. S. Höing, ${ }^{41}$ A. Junkes, ${ }^{41}$ R. Klanner, ${ }^{41}$ R. Kogler, ${ }^{41}$ N. Kovalchuk, ${ }^{41}$ T. Lapsien, ${ }^{41}$ T. Lenz, ${ }^{41}$ I. Marchesini, ${ }^{41}$ D. Marconi, ${ }^{41}$ M. Meyer, ${ }^{41}$ M. Niedziela, ${ }^{41}$ D. Nowatschin, ${ }^{41}$ J. Ott ${ }^{41}$ F. Pantaleo, ${ }^{41, n}$ T. Peiffer, ${ }^{41}$ A. Perieanu, ${ }^{41}$ N. Pietsch, ${ }^{41}$ J. Poehlsen, ${ }^{41}$ C. Sander ${ }^{41}$ C. Scharf ${ }^{41}$ P. Schleper, ${ }^{41}$ E. Schlieckau, ${ }^{41}$ A. Schmidt, ${ }^{41}$ S. Schumann, ${ }^{41}$ J. Schwandt ${ }^{41}$ H. Stadie, ${ }^{41}$ G. Steinbrück,${ }^{41}$ F. M. Stober, ${ }^{41}$ M. Stöver, ${ }^{41}$ H. Tholen, ${ }^{41}$ D. Troendle, ${ }^{41}$ E. Usai, ${ }^{41}$ L. Vanelderen, ${ }^{41}$ A. Vanhoefer ${ }^{41}$ B. Vormwald, ${ }^{41}$ C. Barth,${ }^{42}$ C. Baus, ${ }^{42}$ J. Berger, ${ }^{42}$ E. Butz, ${ }^{42}$ T. Chwalek, ${ }^{42}$ F. Colombo, ${ }^{42}$ W. De Boer, ${ }^{42}$ A. Dierlamm, ${ }^{42}$ S. Fink,${ }^{42}$ R. Friese, ${ }^{42}$ M. Giffels, ${ }^{42}$ A. Gilbert, ${ }^{42}$ D. Haitz, ${ }^{42}$ F. Hartmann,${ }^{42, n}$ S. M. Heindl, ${ }^{42}$ U. Husemann, ${ }^{42}$ I. Katkov, ${ }^{42, o}$ A. Kornmayer, ${ }^{42, \mathrm{n}}$ P. Lobelle Pardo, ${ }^{42}$ B. Maier, ${ }^{42}$ H. Mildner, ${ }^{42}$ M. U. Mozer, ${ }^{42}$ T. Müller, ${ }^{42}$ Th. Müller, ${ }^{42}$ M. Plagge, ${ }^{42}$ G. Quast, ${ }^{42}$ K. Rabbertz, ${ }^{42}$ S. Röcker, ${ }^{42}$ F. Roscher, ${ }^{42}$ M. Schröder, ${ }^{42}$ G. Sieber, ${ }^{42}$ H. J. Simonis ${ }^{42}$ R. Ulrich, ${ }^{42}$ J. Wagner-Kuhr, ${ }^{42}$ S. Wayand, ${ }^{42}$ M. Weber, ${ }^{42}$ T. Weiler ${ }^{42}$ S. Williamson, ${ }^{42}$ C. Wöhrmann, ${ }^{42}$ R. Wolf, ${ }^{42}$ 
G. Anagnostou, ${ }^{43}$ G. Daskalakis ${ }^{43}$ T. Geralis, ${ }^{43}$ V. A. Giakoumopoulou, ${ }^{43}$ A. Kyriakis, ${ }^{43}$ D. Loukas, ${ }^{43}$ I. Topsis-Giotis ${ }^{43}$ A. Agapitos ${ }^{44}$ S. Kesisoglou, ${ }^{44}$ A. Panagiotou, ${ }^{44}$ N. Saoulidou, ${ }^{44}$ E. Tziaferi, ${ }^{44}$ I. Evangelou, ${ }^{45}$ G. Flouris, ${ }^{45}$ C. Foudas, ${ }^{45}$ P. Kokkas, ${ }^{45}$ N. Loukas,${ }^{45}$ N. Manthos ${ }^{45}$ I. Papadopoulos, ${ }^{45}$ E. Paradas ${ }^{45}$ N. Filipovic, ${ }^{46}$ G. Bencze,${ }^{47}$ C. Hajdu ${ }^{47}$ P. Hidas, ${ }^{47}$ D. Horvath, ${ }^{47, t}$ F. Sikler, ${ }^{47}$ V. Veszpremi, ${ }^{47}$ G. Vesztergombi,${ }^{47, u}$ A. J. Zsigmond, ${ }^{47}$ N. Beni ${ }^{48}$ S. Czellar ${ }^{48}$ J. Karancsii, ${ }^{48, v}$ J. Molnar, ${ }^{48}$ Z. Szillasi, ${ }^{48}$ M. Bartók, ${ }^{49, u}$ A. Makovec, ${ }^{49}$ P. Raics, ${ }^{49}$ Z. L. Trocsanyi, ${ }^{49}$ B. Ujvari, ${ }^{49}$

S. Bahinipati, ${ }^{50}$ S. Choudhury, ${ }^{50, w}$ P. Mal,${ }^{50}$ K. Mandal, ${ }^{50}$ A. Nayak,${ }^{50}$ D. K. Sahoo ${ }^{50}$ N. Sahoo, ${ }^{50}$ S. K. Swain, ${ }^{50}$ S. Bansal, ${ }^{51}$ S. B. Beri, ${ }^{51}$ V. Bhatnagar,${ }^{51}$ R. Chawla,${ }^{51}$ R. Gupta,${ }^{51}$ U. Bhawandeep,${ }^{51}$ A. K. Kalsi, ${ }^{51}$ A. Kaur, ${ }^{51}$ M. Kaur, ${ }^{51}$ R. Kumar, ${ }^{51}$ A. Mehta, ${ }^{51}$ M. Mittal, ${ }^{51}$ J. B. Singh,${ }^{51}$ G. Walia, ${ }^{51}$ Ashok Kumar ${ }^{52}$ A. Bhardwaj, ${ }^{52}$ B. C. Choudhary, ${ }^{52}$ R. B. Garg, ${ }^{52}$ S. Keshri, ${ }^{52}$ A. Kumar, ${ }^{52}$ S. Malhotra, ${ }^{52}$ M. Naimuddin, ${ }^{52}$ N. Nishu, ${ }^{52}$ K. Ranjan, ${ }^{52}$ R. Sharma, ${ }^{52}$ V. Sharma ${ }^{52}$ R. Bhattacharya, ${ }^{53}$ S. Bhattacharya, ${ }^{53}$ K. Chatterjee, ${ }^{53}$ S. Dey, ${ }^{53}$ S. Dutt,${ }^{53}$ S. Dutta, ${ }^{53}$ S. Ghosh, ${ }^{53}$ N. Majumdar, ${ }^{53}$ A. Modak,${ }^{53}$ K. Mondal, ${ }^{53}$ S. Mukhopadhyay ${ }^{53}$ S. Nandan, ${ }^{53}$ A. Purohit, ${ }^{53}$ A. Roy, ${ }^{53}$ D. Roy, ${ }^{53}$ S. Roy Chowdhury ${ }^{53}$ S. Sarkar, ${ }^{53}$ M. Sharan, ${ }^{53}$ S. Thakur, ${ }^{53}$ P. K. Behera ${ }^{54}$ R. Chudasama, ${ }^{55}$ D. Dutta,${ }^{55}$ V. Jha, ${ }^{55}$ V. Kumar, ${ }^{55}$ A. K. Mohanty,${ }^{55, n}$ P. K. Netrakanti ${ }^{55}$ L. M. Pant, ${ }^{55}$ P. Shukla ${ }^{55}$ A. Topkar, ${ }^{55}$ T. Aziz,${ }^{56}$ S. Banerjee, ${ }^{56}$ S. Bhowmik, ${ }^{56, x}$ R. M. Chatterjee,${ }^{56}$ R. K. Dewanjee, ${ }^{56}$ S. Dugad, ${ }^{56}$ S. Ganguly, ${ }^{56}$ M. Guchait,${ }^{56}$ A. Gurtu, ${ }^{56, y}$ Sa. Jain, ${ }^{56}$ G. Kole, ${ }^{56}$ S. Kumar, ${ }^{56}$ B. Mahakud, ${ }^{56}$ M. Maity, ${ }^{56, x}$ G. Majumder, ${ }^{56}$ K. Mazumdar, ${ }^{56}$ S. Mitra ${ }^{56}$ G. B. Mohanty,${ }^{56}$ B. Parida,${ }^{56}$ T. Sarkar, ${ }^{56, x}$ N. Sur, ${ }^{56}$ B. Sutar,${ }^{56}$ N. Wickramage, ${ }^{56, z}$ S. Chauhan, ${ }^{57}$ S. Dube, ${ }^{57}$ A. Kapoor,${ }^{57}$ K. Kothekar, ${ }^{57}$ A. Rane, ${ }^{57}$ S. Sharma, ${ }^{57}$ H. Bakhshiansohi,${ }^{58}$

H. Behnamian, ${ }^{58}$ S. Chenarani, ${ }^{58}$ E. Eskandari Tadavani, ${ }^{58}$ S. M. Etesami, ${ }^{58, \text { aa }}$ A. Fahim, ${ }^{58, b b}$ M. Khakzad, ${ }^{58}$

M. Mohammadi Najafabadi, ${ }^{58}$ M. Naseri, ${ }^{58}$ S. Paktinat Mehdiabadi, ${ }^{58}$ F. Rezaei Hosseinabadi, ${ }^{58}$ B. Safarzadeh, ${ }^{58, c c}$ M. Zeinali ${ }^{58}$ M. Felcini, ${ }^{59}$ M. Grunewald, ${ }^{59}$ M. Abbrescia, ${ }^{60 a, 60 b}$ C. Calabria, ${ }^{60 a, 60 b}$ C. Caputo, ${ }^{60 a, 60 b}$ A. Colaleo, ${ }^{60 a}$ D. Creanza,${ }^{60 a, 60 c}$ L. Cristella, ${ }^{60 a, 60 b}$ N. De Filippis, ${ }^{60 a, 60 c}$ M. De Palma,${ }^{60 a, 60 b}$ L. Fiore, ${ }^{60 a}$ G. Iaselli, ${ }^{60 a, 60 c}$ G. Maggi, ${ }^{60 a, 60 c}$ M. Maggi, ${ }^{60 \mathrm{a}}$ G. Miniello, ${ }^{60 \mathrm{a}, 60 \mathrm{~b}}$ S. My, ${ }^{60 \mathrm{a}, 60 \mathrm{~b}}$ S. Nuzzo, ${ }^{60 \mathrm{a}, 60 \mathrm{~b}}$ A. Pompili, ${ }^{60 \mathrm{a}, 60 \mathrm{~b}}$ G. Pugliese, ${ }^{60 \mathrm{a}, 60 \mathrm{c}}$ R. Radogna, ${ }^{60 \mathrm{a}, 60 \mathrm{~b}}$ A. Ranieri, ${ }^{60 \mathrm{a}}$ G. Selvaggi, ${ }^{60 a, 60 \mathrm{~b}}$ L. Silvestris, ${ }^{60 a, n}$ R. Venditti, ${ }^{60,60 \mathrm{~b}}$ G. Abbiendi, ${ }^{61 \mathrm{a}}$ C. Battilana,${ }^{61 \mathrm{a}}$ D. Bonacorsi, ${ }^{61 \mathrm{a}, 61 \mathrm{~b}}$ S. Braibant-Giacomelli, ${ }^{61 \mathrm{a}, 61 \mathrm{~b}}$ L. Brigliadori, ${ }^{61 \mathrm{a}, 61 \mathrm{~b}}$ R. Campanini, ${ }^{61 \mathrm{a}, 61 \mathrm{~b}}$ P. Capiluppi, ${ }^{61 \mathrm{a}, 61 \mathrm{~b}}$ A. Castro, ${ }^{61 \mathrm{a}, 61 \mathrm{~b}}$ F. R. Cavallo, ${ }^{61 \mathrm{a}}$ S. S. Chhibra, ${ }^{61 a, 61 b}$ G. Codispoti, ${ }^{61 a, 61 b}$ M. Cuffiani, ${ }^{61 a, 61 b}$ G. M. Dallavalle, ${ }^{61 a}$ F. Fabbri, ${ }^{61 a}$ A. Fanfani, ${ }^{61 a, 61 b}$ D. Fasanella, ${ }^{61 a, 61 b}$ P. Giacomelli, ${ }^{61 a}$ C. Grandi, ${ }^{61 a}$ L. Guiducci, ${ }^{61 a, 61 b}$ S. Marcellini, ${ }^{61 a}$ G. Masetti, ${ }^{61 a}$ A. Montanari, ${ }^{61 a}$ F. L. Navarria, ${ }^{61 a, 61 b}$ A. Perrotta, ${ }^{61 a}$ A. M. Rossi, ${ }^{61 a, 61 b}$ T. Rovelli, ${ }^{61 a, 61 b}$ G. P. Siroli, ${ }^{61 a, 61 b}$ N. Tosi, ${ }^{61 a, 61 b, n}$ S. Albergo, ${ }^{62 a, 62 b}$ M. Chiorboli, ${ }^{62 a, 62 b}$ S. Costa, ${ }^{62 a, 62 b}$ A. Di Mattia, ${ }^{62 a}$ F. Giordano, ${ }^{62 a, 62 b}$ R. Potenza,${ }^{62 a, 62 b}$ A. Tricomi, ${ }^{62 a, 62 b}$ C. Tuve, ${ }^{62 a, 62 b}$ G. Barbagli, ${ }^{63 a}$ V. Ciulli, ${ }^{63 a, 63 b}$ C. Civinini, ${ }^{63 a}$ R. D’Alessandro, ${ }^{63 a, 63 b}$ E. Focardi, ${ }^{63 a, 63 b}$ V. Gori, ${ }^{63 a, 63 b}$ P. Lenzi, ${ }^{63 a, 63 b}$ M. Meschini, ${ }^{63 a}$ S. Paoletti, ${ }^{63 a}$ G. Sguazzoni, ${ }^{63 a}$ L. Viliani,,${ }^{63 a, 63 b, n}$ L. Benussi, ${ }^{64}$ S. Bianco, ${ }^{64}$ F. Fabbri, ${ }^{64}$ D. Piccolo, ${ }^{64}$ F. Primavera, ${ }^{64, n}$ V. Calvelli, ${ }^{65 a, 65 b}$ F. Ferro, ${ }^{65 a}$ M. Lo Vetere, ${ }^{65 a, 65 b}$ M. R. Monge, ${ }^{65 a, 65 b}$ E. Robutti, ${ }^{65 a}$ S. Tosi, ${ }^{65 a, 65 b}$ L. Brianza, ${ }^{66 \mathrm{a}}$ F. Brivio, ${ }^{66 \mathrm{a}}$ M. E. Dinardo ${ }^{66 \mathrm{a}, 66 \mathrm{~b}}$ S. Fiorendi, ${ }^{66 a, 66 \mathrm{~b}}$ S. Gennai, ${ }^{66 \mathrm{a}}$ A. Ghezzi, ${ }^{66 \mathrm{a}, 66 \mathrm{~b}}$ P. Govoni, ${ }^{66 a, 66 \mathrm{~b}}$ S. Malvezzi, ${ }^{66 \mathrm{a}}$ R. A. Manzoni, ${ }^{66 a, 66 \mathrm{~b}, \mathrm{n}}$ B. Marzocchi, ${ }^{66 \mathrm{a}, 66 \mathrm{~b}}$ D. Menasce, ${ }^{66 \mathrm{a}}$ L. Moroni, ${ }^{66 \mathrm{a}}$ M. Paganoni, ${ }^{66 \mathrm{a}, 66 \mathrm{~b}}$ D. Pedrini, ${ }^{66 \mathrm{a}}$

S. Pigazzini, ${ }^{66 \mathrm{a}}$ S. Ragazzi, ${ }^{66 a, 66 \mathrm{~b}}$ T. Tabarelli de Fatis, ${ }^{66 a, 66 \mathrm{~b}}$ S. Buontempo, ${ }^{67 \mathrm{a}}$ N. Cavallo, ${ }^{67 a, 67 \mathrm{c}}$ G. De Nardo, ${ }^{67 \mathrm{a}}$ S. Di Guida, ${ }^{67 a, 67 \mathrm{~d}, \mathrm{n}}$ M. Esposito, ${ }^{67 a, 67 b}$ F. Fabozzi, ${ }^{67 a, 67 \mathrm{c}}$ A. O. M. Iorio, ${ }^{67 a, 67 b}$ G. Lanza, ${ }^{67 a}$ L. Lista, ${ }^{67 a}$ S. Meola, ${ }^{67 a, 67 \mathrm{~d}, \mathrm{n}}$ M. Merola,${ }^{67 \mathrm{a}}$ P. Paolucci, ${ }^{67 a, \mathrm{n}}$ C. Sciacca, ${ }^{67 a, 67 \mathrm{~b}}$ F. Thyssen,${ }^{67 \mathrm{a}}$ P. Azzi, ${ }^{68 a, \mathrm{n}}$ N. Bacchetta, ${ }^{68 \mathrm{a}}$ L. Benato, ${ }^{68 \mathrm{a}, 68 \mathrm{~b}}$ D. Bisello, ${ }^{68 \mathrm{a}, 68 \mathrm{~b}}$ A. Boletti, ${ }^{68 a, 68 b}$ R. Carlin, ${ }^{68 a, 68 b}$ A. Carvalho Antunes De Oliveira, ${ }^{68 a, 68 b}$ P. Checchia, ${ }^{68 \mathrm{a}}$ M. Dall'Osso, ${ }^{68 a, 68 b}$ P. De Castro Manzano, ${ }^{68 \mathrm{a}}$ T. Dorigo, ${ }^{68 \mathrm{a}}$ U. Dosselli, ${ }^{68 \mathrm{a}}$ F. Gasparini, ${ }^{68,68 \mathrm{~b}}$ U. Gasparini, ${ }^{68 \mathrm{a}, 68 \mathrm{~b}}$ A. Gozzelino, ${ }^{68 \mathrm{a}}$ S. Lacaprara, ${ }^{68 \mathrm{a}}$ M. Margoni, ${ }^{68,68 \mathrm{~b}}$ A. T. Meneguzzo, ${ }^{68 \mathrm{a}, 68 \mathrm{~b}}$ J. Pazzini, ${ }^{68 \mathrm{a}, 68 \mathrm{~b}, \mathrm{n}}$ N. Pozzobon, ${ }^{68 \mathrm{a}, 68 \mathrm{~b}}$ P. Ronchese, ${ }^{68 \mathrm{a}, 68 \mathrm{~b}}$ F. Simonetto, ${ }^{68 a, 68 b}$ E. Torassa, ${ }^{68 \mathrm{a}}$ M. Tosi, ${ }^{68 a, 68 b}$ M. Zanetti, ${ }^{68 \mathrm{a}}$ P. Zotto, ${ }^{68 a, 68 b}$ A. Zucchetta, ${ }^{68 \mathrm{a}, 68 \mathrm{~b}}$ G. Zumerle, ${ }^{68 \mathrm{a}, 68 \mathrm{~b}}$ A. Braghieri, ${ }^{69 a}$ A. Magnani, ${ }^{6 a, 69 b}$ P. Montagna,${ }^{69 a, 69 b}$ S. P. Ratti, ${ }^{69 a, 69 b}$ V. Re ${ }^{69 a}$ C. Riccardi, ${ }^{69 a, 69 b}$ P. Salvini,${ }^{69 a}$ I. Vai, ${ }^{69 a, 69 b}$ P. Vitulo, ${ }^{69 a, 69 b}$ L. Alunni Solestizi, ${ }^{70 a, 70 b}$ G. M. Bilei, ${ }^{70 a}$ D. Ciangottini, ${ }^{70 a, 70 b}$ L. Fanò, ${ }^{70 a, 70 b}$ P. Lariccia, ${ }^{70 a, 70 b}$ R. Leonardi, ${ }^{70 a, 70 b}$ G. Mantovani, ${ }^{70 a, 70 b}$ M. Menichellii, ${ }^{70 a}$ A. Saha, ${ }^{70 a}$ A. Santocchia, ${ }^{70 a, 70 b}$ K. Androsov, ${ }^{71 a, d d}$ P. Azzurri, ${ }^{71 a, n}$ G. Bagliesi, ${ }^{71 a}$ J. Bernardini, ${ }^{71 \mathrm{a}}$ T. Boccali, ${ }^{71 \mathrm{a}}$ R. Castaldi, ${ }^{71 \mathrm{a}}$ M. A. Ciocci, ${ }^{71 \mathrm{a}, \mathrm{dd}}$ R. Dell'Orso, ${ }^{71 \mathrm{a}}$ S. Donato, ${ }^{71 \mathrm{a}, 71 \mathrm{c}}$ G. Fedi, ${ }^{71 \mathrm{a}}$ A. Giassi ${ }^{71 \mathrm{a}}$ M. T. Grippo, ${ }^{71 \mathrm{a}, \mathrm{dd}}$ F. Ligabue, ${ }^{71 \mathrm{a}, 7 \mathrm{c}}$ T. Lomtadze, ${ }^{71 \mathrm{a}}$ L. Martini, ${ }^{71 \mathrm{a}, 71 \mathrm{~b}}$ A. Messineo, ${ }^{71 \mathrm{a}, 71 \mathrm{~b}}$ F. Palla, ${ }^{71 \mathrm{a}}$ A. Rizzi, ${ }^{71,71 b}$ A. Savoy-Navarro, ${ }^{71 a, e e}$ P. Spagnolo, ${ }^{71 a}$ R. Tenchini, ${ }^{71 a}$ G. Tonelli, ${ }^{71 a, 71 b}$ A. Venturi, ${ }^{71 a}$ P. G. Verdini, ${ }^{71 a}$ L. Barone,${ }^{72 a, 72 b}$ F. Cavallari, ${ }^{72 a}$ M. Cipriani, ${ }^{72 a, 72 b}$ G. D'imperio, ${ }^{72 a, 72 b, n}$ D. Del Re ${ }^{72 a, 72 b, n}$ M. Diemoz, ${ }^{72 a}$ S. Gelli, ${ }^{72 a, 72 b}$ C. Jorda, ${ }^{72 a}$ E. Longo, ${ }^{72 a, 72 b}$ F. Margaroli, ${ }^{72 a, 72 b}$ P. Meridiani, ${ }^{72 a}$ G. Organtini, ${ }^{72 a, 72 b}$ R. Paramatti, ${ }^{72 a}$ F. Preiato, ${ }^{72 a, 72 b}$ S. Rahatlou, ${ }^{72 a, 72 b}$ C. Rovelli, ${ }^{72 a}$ F. Santanastasio, ${ }^{72 a, 72 b}$ N. Amapane, ${ }^{73 a, 73 b}$ R. Arcidiacono, ${ }^{73 a, 73 c, n}$ S. Argiro, ${ }^{73 a, 73 b}$ M. Arneodo, ${ }^{73 a, 73 c}$ N. Bartosik, ${ }^{73 a}$ R. Bellan, ${ }^{73 a, 73 b}$ C. Biino, ${ }^{73 a}$ N. Cartiglia, ${ }^{73 a}$ M. Costa, ${ }^{73 a, 73 b}$ R. Covarelli, ${ }^{73 a, 73 b}$ 
A. Degano, ${ }^{73 a, 73 b}$ N. Demaria, ${ }^{73 a}$ L. Finco, ${ }^{73 a, 73 b}$ B. Kiani, ${ }^{73 a, 73 b}$ C. Mariotti, ${ }^{73 a}$ S. Maselli, ${ }^{73 a}$ E. Migliore, ${ }^{73 a, 73 b}$ V. Monaco, ${ }^{73 a, 73 b}$ E. Monteil,${ }^{73 a, 73 b}$ M. M. Obertino, ${ }^{73 a, 73 b}$ L. Pacher, ${ }^{73 a, 73 b}$ N. Pastrone, ${ }^{73 a}$ M. Pelliccioni, ${ }^{73 a}$ G. L. Pinna Angioni, ${ }^{73 a, 73 b}$ F. Ravera, ${ }^{73 a, 73 b}$ A. Romero, ${ }^{73 a, 73 b}$ M. Ruspa ${ }^{73 a, 73 c}$ R. Sacchi, ${ }^{73 a, 73 b}$ V. Sola, ${ }^{73 a}$ A. Solano, ${ }^{73 a, 73 b}$ A. Staiano, ${ }^{73 a}$ P. Traczyk, ${ }^{73 a, 73 b}$ S. Belforte, ${ }^{74 a}$ V. Candelise,${ }^{74 a, 74 b}$ M. Casarsa, ${ }^{74 a}$ F. Cossutti, ${ }^{74 a}$ G. Della Ricca, ${ }^{74 a, 74 b}$ C. La Licata, ${ }^{74 a, 74 b}$ A. Schizzi, ${ }^{74 a, 74 b}$ A. Zanetti, ${ }^{74 a}$ D. H. Kim,${ }^{75}$ G. N. Kim,${ }^{75}$ M. S. Kim, ${ }^{75}$ S. Lee, ${ }^{75}$ S. W. Lee, ${ }^{75}$ Y. D. Oh, ${ }^{75}$ S. Sekmen ${ }^{75}$ D. C. Son ${ }^{75}$ Y. C. Yang,${ }^{75}$ H. Kim,${ }^{76}$ J. A. Brochero Cifuentes,${ }^{77}$ T. J. Kim,${ }^{77}$ S. Cho, ${ }^{78}$ S. Choi, ${ }^{78}$ Y. Go ${ }^{78}$ D. Gyun, ${ }^{78}$ S. Ha, ${ }^{78}$ B. Hong, ${ }^{78}$ Y. Jo ${ }^{78}$ Y. Kim, ${ }^{78}$ B. Lee, ${ }^{78}$ K. Lee, ${ }^{78}$ K. S. Lee ${ }^{78}$ S. Lee ${ }^{78}$ J. Lim, ${ }^{78}$ S. K. Park, ${ }^{78}$ Y. Roh ${ }^{78}$ J. Almond ${ }^{79}$ J. Kim, ${ }^{79}$ S. H. Seo, ${ }^{79}$ U. K. Yang, ${ }^{79}$ H. D. Yoo, ${ }^{79}$ G. B. Yu ${ }^{79}$ M. Choi, ${ }^{80}$ H. Kim,${ }^{80}$ H. Kim,${ }^{80}$ J. H. Kim,${ }^{80}$ J. S. H. Lee, ${ }^{80}$ I. C. Park, ${ }^{80}$ G. Ryu, ${ }^{80}$ M. S. Ryu, ${ }^{80}$ Y. Choi, ${ }^{81}$ J. Goh, ${ }^{81}$ D. Kim,${ }^{81}$ E. Kwon, ${ }^{81}$ J. Lee, ${ }^{81}$ I. Yu, ${ }^{81}$ V. Dudenas, ${ }^{82}$ A. Juodagalvis, ${ }^{82}$ J. Vaitkus, ${ }^{82}$ I. Ahmed ${ }^{83}$ Z. A. Ibrahim, ${ }^{83}$ J. R. Komaragiri, ${ }^{83}$ M. A. B. Md Ali, ${ }^{83, f f}$ F. Mohamad Idris,,${ }^{83, g g}$ W. A. T. Wan Abdullah, ${ }^{83}$ M. N. Yusli, ${ }^{83}$ Z. Zolkapli ${ }^{83}$ E. Casimiro Linares, ${ }^{84}$ H. Castilla-Valdez,${ }^{84}$ E. De La Cruz-Burelo, ${ }^{84}$ I. Heredia-De La Cruz, ${ }^{84, \text { hh }}$ A. Hernandez-Almada,${ }^{84}$ R. Lopez-Fernandez, ${ }^{84}$ J. Mejia Guisao, ${ }^{84}$ A. Sanchez-Hernandez, ${ }^{84}$ S. Carrillo Moreno, ${ }^{85}$ F. Vazquez Valencia ${ }^{85}$ I. Pedraza,${ }^{86}$ H. A. Salazar Ibarguen, ${ }^{86}$ C. Uribe Estrada, ${ }^{86}$

A. Morelos Pineda, ${ }^{87}$ D. Krofcheck, ${ }^{88}$ P. H. Butler, ${ }^{89}$ A. Ahmad,${ }^{90}$ M. Ahmad, ${ }^{90}$ Q. Hassan, ${ }^{90}$ H. R. Hoorani, ${ }^{90}$ W. A. Khan, ${ }^{90}$ T. Khurshid, ${ }^{90}$ M. Shoaib, ${ }^{90}$ M. Waqas, ${ }^{90}$ H. Bialkowska, ${ }^{91}$ M. Bluj, ${ }^{91}$ B. Boimska, ${ }^{91}$ T. Frueboes,${ }^{91}$ M. Górski, ${ }^{91}$ M. Kazana, ${ }^{91}$ K. Nawrocki,${ }^{91}$ K. Romanowska-Rybinska, ${ }^{91}$ M. Szleper, ${ }^{91}$ P. Zalewski, ${ }^{91}$ K. Bunkowski, ${ }^{92}$ A. Byszuk, ${ }^{92, i i}$ K. Doroba,${ }^{92}$ A. Kalinowski, ${ }^{92}$ M. Konecki, ${ }^{92}$ J. Krolikowski,${ }^{92}$ M. Misiura, ${ }^{92}$ M. Olszewski,${ }^{92}$ M. Walczak,${ }^{92}$ P. Bargassa, ${ }^{93}$ C. Beirão Da Cruz E Silva, ${ }^{93}$ A. Di Francesco, ${ }^{93}$ P. Faccioli ${ }^{93}$ P. G. Ferreira Parracho,${ }^{93}$ M. Gallinaro, ${ }^{93}$ J. Hollar, ${ }^{93}$ N. Leonardo, ${ }^{93}$ L. Lloret Iglesias, ${ }^{93}$ M. V. Nemallapudi, ${ }^{93}$ F. Nguyen, ${ }^{93}$ J. Rodrigues Antunes, ${ }^{93}$ J. Seixas, ${ }^{93}$ O. Toldaiev, ${ }^{93}$ D. Vadruccio, ${ }^{93}$ J. Varela,${ }^{93}$ P. Vischia,${ }^{93}$ P. Bunin, ${ }^{94}$ I. Golutvin, ${ }^{94}$ N. Gorbounov, ${ }^{94}$ I. Gorbunov, ${ }^{94}$ A. Kamenev, ${ }^{94}$ V. Karjavin, ${ }^{94}$ A. Lanev, ${ }^{94}$ A. Malakhov, ${ }^{94}$ V. Matveev, ${ }^{94, j j, k k}$ P. Moisenz, ${ }^{94}$ V. Palichik, ${ }^{94}$ V. Perelygin, ${ }^{94}$ M. Savina, ${ }^{94}$ S. Shmatov, ${ }^{94}$ S. Shulha,${ }^{94}$ N. Skatchkov, ${ }^{94}$ V. Smirnov, ${ }^{94}$ N. Voytishin, ${ }^{94}$ A. Zarubin,${ }^{94}$ L. Chtchipounov, ${ }^{95}$ V. Golovtsov, ${ }^{95}$ Y. Ivanov, ${ }^{95}$ V. Kim,${ }^{95,11}$ E. Kuznetsova, ${ }^{95, m m}$ V. Murzin, ${ }^{95}$ V. Oreshkin, ${ }^{95}$ V. Sulimov, ${ }^{95}$ A. Vorobyev, ${ }^{95}$ Yu. Andreev, ${ }^{96}$ A. Dermenev, ${ }^{96}$ S. Gninenko,${ }^{96}$ N. Golubev, ${ }^{96}$ A. Karneyeu, ${ }^{96}$ M. Kirsanov, ${ }^{96}$ N. Krasnikov, ${ }^{96}$ A. Pashenkov, ${ }^{96}$ D. Tlisov, ${ }^{96}$ A. Toropin, ${ }^{96}$ V. Epshteyn, ${ }^{97}$ V. Gavrilov,${ }^{97}$ N. Lychkovskaya,${ }^{97}$ V. Popov,${ }^{97}$ I. Pozdnyakov, ${ }^{97}$ G. Safronov, ${ }^{97}$ A. Spiridonov, ${ }^{97}$ M. Toms,${ }^{97}$ E. Vlasov, ${ }^{97}$ A. Zhokin, ${ }^{97}$ R. Chistov, ${ }^{98}$ M. Danilov, ${ }^{98}$ V. Rusinov, ${ }^{98}$ V. Andreev, ${ }^{99}$ M. Azarkin, ${ }^{99, k \mathrm{k}}$ I. Dremin, ${ }^{99, \mathrm{kk}}$ M. Kirakosyan, ${ }^{99}$ A. Leonidov, ${ }^{99, \mathrm{kk}}$ S. V. Rusakov, ${ }^{99}$ A. Terkulov, ${ }^{99}$ A. Baskakov, ${ }^{100}$ A. Belyaev,${ }^{100}$ E. Boos,${ }^{100}$ M. Dubinin, ${ }^{100, n n}$ L. Dudko, ${ }^{100}$ A. Ershov, ${ }^{100}$ A. Gribushin, ${ }^{100}$ V. Klyukhin, ${ }^{100}$ O. Kodolova, ${ }^{100}$ I. Lokhtin, ${ }^{100}$ I. Miagkov, ${ }^{100}$ S. Obraztsov, ${ }^{100}$ S. Petrushanko, ${ }^{100}$ V. Savrin, ${ }^{100}$ A. Snigirev, ${ }^{100}$ I. Azhgirey, ${ }^{101}$ I. Bayshev, ${ }^{101}$ S. Bitioukov, ${ }^{101}$ D. Elumakhov, ${ }^{101}$ V. Kachanov, ${ }^{101}$ A. Kalinin, ${ }^{101}$ D. Konstantinov, ${ }^{101}$ V. Krychkine, ${ }^{101}$ V. Petrov, ${ }^{101}$ R. Ryutin, ${ }^{101}$ A. Sobol, ${ }^{101}$ S. Troshin, ${ }^{101}$ N. Tyurin, ${ }^{101}$ A. Uzunian, ${ }^{101}$ A. Volkov, ${ }^{101}$ P. Adzic, ${ }^{102,0 o}$ P. Cirkovic, ${ }^{102}$ D. Devetak, ${ }^{102}$ J. Milosevic, ${ }^{102}$ V. Rekovic, ${ }^{102}$ J. Alcaraz Maestre, ${ }^{103}$ E. Calvo,${ }^{103}$ M. Cerrada,${ }^{103}$

M. Chamizo Llatas, ${ }^{103}$ N. Colino, ${ }^{103}$ B. De La Cruz,${ }^{103}$ A. Delgado Peris, ${ }^{103}$ A. Escalante Del Valle, ${ }^{103}$ C. Fernandez Bedoya, ${ }^{103}$ J. P. Fernández Ramos, ${ }^{103}$ J. Flix, ${ }^{103}$ M. C. Fouz, ${ }^{103}$ P. Garcia-Abia, ${ }^{103}$ O. Gonzalez Lopez, ${ }^{103}$ S. Goy Lopez, ${ }^{103}$ J. M. Hernandez, ${ }^{103}$ M. I. Josa,${ }^{103}$ E. Navarro De Martino, ${ }^{103}$ A. Pérez-Calero Yzquierdo, ${ }^{103}$ J. Puerta Pelayo, ${ }^{103}$ A. Quintario Olmeda, ${ }^{103}$ I. Redondo, ${ }^{103}$ L. Romero, ${ }^{103}$ M. S. Soares, ${ }^{103}$ J. F. de Trocóniz, ${ }^{104}$ M. Missiroli, ${ }^{104}$ D. Moran, ${ }^{104}$ J. Cuevas, ${ }^{105}$ J. Fernandez Menendez, ${ }^{105}$ I. Gonzalez Caballero, ${ }^{105}$ E. Palencia Cortezon, ${ }^{105}$ S. Sanchez Cruz, ${ }^{105}$ J. M. Vizan Garcia, ${ }^{105}$ I. J. Cabrillo, ${ }^{106}$ A. Calderon, ${ }^{106}$ J. R. Castiñeiras De Saa, ${ }^{106}$ E. Curras, ${ }^{106}$ M. Fernandez, ${ }^{106}$ J. Garcia-Ferrero, ${ }^{106}$ G. Gomez, ${ }^{106}$ A. Lopez Virto, ${ }^{106}$ J. Marco,${ }^{106}$ C. Martinez Rivero, ${ }^{106}$ F. Matorras, ${ }^{106}$ J. Piedra Gomez, ${ }^{106}$ T. Rodrigo, ${ }^{106}$ A. Ruiz-Jimeno, ${ }^{106}$ L. Scodellaro, ${ }^{106}$ N. Trevisani, ${ }^{106}$ I. Vila, ${ }^{106}$ R. Vilar Cortabitarte, ${ }^{106}$ D. Abbaneo, ${ }^{107}$ E. Auffray, ${ }^{107}$ G. Auzinger, ${ }^{107}$ M. Bachtis, ${ }^{107}$ P. Baillon, ${ }^{107}$ A. H. Ball, ${ }^{107}$ D. Barney, ${ }^{107}$ P. Bloch, ${ }^{107}$ A. Bocci, ${ }^{107}$ A. Bonato, ${ }^{107}$ C. Botta, ${ }^{107}$ T. Camporesi,${ }^{107}$ R. Castello, ${ }^{107}$ M. Cepeda,${ }^{107}$ G. Cerminara,${ }^{107}$ M. D' Alfonso, ${ }^{107}$ D. d'Enterria ${ }^{107}$ A. Dabrowski, ${ }^{107}$ V. Daponte, ${ }^{107}$ A. David, ${ }^{107}$ M. De Gruttola,${ }^{107}$ F. De Guio, ${ }^{107}$ A. De Roeck,${ }^{107}$ E. Di Marco, ${ }^{107, p p}$ M. Dobson, ${ }^{107}$ M. Dordevic, ${ }^{107}$ B. Dorney, ${ }^{107}$ T. du Pree,${ }^{107}$ D. Duggan, ${ }^{107}$ M. Dünser, ${ }^{107}$ N. Dupont, ${ }^{107}$ A. Elliott-Peisert, ${ }^{107}$ S. Fartoukh, ${ }^{107}$ G. Franzoni, ${ }^{107}$ J. Fulcher, ${ }^{107}$ W. Funk, ${ }^{107}$ D. Gigi, ${ }^{107}$ K. Gill, ${ }^{107}$ M. Girone, ${ }^{107}$ F. Glege ${ }^{107}$ S. Gundacker, ${ }^{107}$ M. Guthoff, ${ }^{107}$ J. Hammer, ${ }^{107}$ P. Harris,${ }^{107}$ J. Hegeman, ${ }^{107}$ V. Innocente, ${ }^{107}$ P. Janot,${ }^{107}$ H. Kirschenmann, ${ }^{107}$ V. Knünz, ${ }^{107}$ M. J. Kortelainen, ${ }^{107}$ K. Kousouris, ${ }^{107}$ M. Krammer, ${ }^{107, b}$ P. Lecoq,${ }^{107}$ C. Lourenço, ${ }^{107}$ M. T. Lucchini, ${ }^{107}$ N. Magini, ${ }^{107}$ L. Malgeri, ${ }^{107}$ M. Mannelli, ${ }^{107}$ A. Martelli, ${ }^{107}$ F. Meijers, ${ }^{107}$ S. Mersi, ${ }^{107}$ E. Meschi, ${ }^{107}$ F. Moortgat, ${ }^{107}$ S. Morovic, ${ }^{107}$ M. Mulders, ${ }^{107}$ H. Neugebauer, ${ }^{107}$ S. Orfanelli, ${ }^{107, q 9}$ L. Orsini, ${ }^{107}$ L. Pape,${ }^{107}$ E. Perez, ${ }^{107}$ 
M. Peruzzi, ${ }^{107}$ A. Petrilli, ${ }^{107}$ G. Petrucciani, ${ }^{107}$ A. Pfeiffer,${ }^{107}$ M. Pierini, ${ }^{107}$ A. Racz, ${ }^{107}$ T. Reis, ${ }^{107}$ G. Rolandi, ${ }^{107, \text { rr }}$ M. Rovere, ${ }^{107}$ M. Ruan, ${ }^{107}$ H. Sakulin, ${ }^{107}$ J. B. Sauvan, ${ }^{107}$ C. Schäfer, ${ }^{107}$ C. Schwick, ${ }^{107}$ M. Seidel, ${ }^{107}$ A. Sharma, ${ }^{107}$ P. Silva, ${ }^{107}$ M. Simon, ${ }^{107}$ P. Sphicas, ${ }^{107, s s}$ J. Steggemann, ${ }^{107}$ M. Stoye ${ }^{107}$ Y. Takahashi, ${ }^{107}$ D. Treille, ${ }^{107}$ A. Triossi, ${ }^{107}$ A. Tsirou, ${ }^{107}$ V. Veckalns, ${ }^{107, \text { tt }}$ G. I. Veres, ${ }^{107, \text { u }}$ N. Wardle, ${ }^{107}$ A. Zagozdzinska, ${ }^{107, \text { ii }}$ W. D. Zeuner, ${ }^{107}$ W. Bertl,,${ }^{108}$ K. Deiters, ${ }^{108}$ W. Erdmann, ${ }^{108}$ R. Horisberger, ${ }^{108}$ Q. Ingram, ${ }^{108}$ H. C. Kaestli, ${ }^{108}$ D. Kotlinski, ${ }^{108}$ U. Langenegger, ${ }^{108}$ T. Rohe ${ }^{108}$ F. Bachmair, ${ }^{109}$ L. Bäni, ${ }^{109}$ L. Bianchini, ${ }^{109}$ B. Casal, ${ }^{109}$ G. Dissertori, ${ }^{109}$ M. Dittmar, ${ }^{109}$ M. Donegà, ${ }^{109}$ P. Eller ${ }^{109}$ C. Grab,${ }^{109}$ C. Heidegger ${ }^{109}$ D. Hits,${ }^{109}$ J. Hoss, ${ }^{109}$ G. Kasieczka, ${ }^{109}$ P. Lecomte,${ }^{109, a}$ W. Lustermann, ${ }^{109}$ B. Mangano, ${ }^{109}$ M. Marionneau, ${ }^{109}$ P. Martinez Ruiz del Arbol, ${ }^{109}$ M. Masciovecchio, ${ }^{109}$ M. T. Meinhard, ${ }^{109}$ D. Meister, ${ }^{109}$ F. Micheli, ${ }^{109}$ P. Musella, ${ }^{109}$ F. Nessi-Tedaldi, ${ }^{109}$ F. Pandolfi, ${ }^{109}$ J. Pata, ${ }^{109}$ F. Pauss, ${ }^{109}$ G. Perrin, ${ }^{109}$ L. Perrozzi, ${ }^{109}$ M. Quittnat ${ }^{109}$ M. Rossini, ${ }^{109}$ M. Schönenberger ${ }^{109}$ A. Starodumov, ${ }^{109, \text { uu }}$ M. Takahashi, ${ }^{109}$ V. R. Tavolaro, ${ }^{109}$ K. Theofilatos, ${ }^{109}$ R. Wallny, ${ }^{109}$ T. K. Aarrestad, ${ }^{110}$ C. Amsler, ${ }^{110, v v}$ L. Caminada, ${ }^{110}$ M. F. Canelli, ${ }^{110}$ V. Chiochia, ${ }^{110}$ A. De Cosa,${ }^{110}$ C. Galloni, ${ }^{110}$ A. Hinzmann, ${ }^{110}$ T. Hreus,${ }^{110}$ B. Kilminster,${ }^{110}$ C. Lange,${ }^{110}$ J. Ngadiuba ${ }^{110}$ D. Pinna,${ }^{110}$ G. Rauco, ${ }^{110}$ P. Robmann, ${ }^{110}$ D. Salerno, ${ }^{110}$ Y. Yang,${ }^{110}$ K. H. Chen,${ }^{111}$ T. H. Doan, ${ }^{111}$ Sh. Jain, ${ }^{111}$ R. Khurana, ${ }^{111}$ M. Konyushikhin, ${ }^{111}$ C. M. Kuo, ${ }^{111}$ W. Lin, ${ }^{111}$ Y. J. Lu, ${ }^{111}$ A. Pozdnyakov, ${ }^{111}$ S. S. Yu, ${ }^{111}$ Arun Kumar, ${ }^{112}$ P. Chang, ${ }^{112}$ Y. H. Chang, ${ }^{112}$ Y. W. Chang, ${ }^{112}$ Y. Chao, ${ }^{112}$ K. F. Chen, ${ }^{112}$ P. H. Chen, ${ }^{112}$ C. Dietz, ${ }^{112}$ F. Fiori, ${ }^{112}$ W.-S. Hou, ${ }^{112}$ Y. Hsiung, ${ }^{112}$ Y. F. Liu ${ }^{112}$ R.-S. Lu, ${ }^{112}$ M. Miñano Moya, ${ }^{112}$ E. Paganis,${ }^{112}$ J. f. Tsai, ${ }^{112}$ Y. M. Tzeng, ${ }^{112}$ B. Asavapibhop, ${ }^{113}$ G. Singh, ${ }^{113}$ N. Srimanobhas, ${ }^{113}$ N. Suwonjandee, ${ }^{113}$ A. Adiguzel, ${ }^{114}$ M. N. Bakirci, ${ }^{14, w w}$ S. Damarseckin, ${ }^{114}$ Z. S. Demiroglu ${ }^{114}$ C. Dozen, ${ }^{114}$ I. Dumanoglu, ${ }^{114}$ S. Girgis, ${ }^{114}$ G. Gokbulut, ${ }^{114}$ Y. Guler,${ }^{114}$ E. Gurpinar, ${ }^{114}$ I. Hos, ${ }^{114}$ E. E. Kangal, ${ }^{114, x x}$ A. Kayis Topaksu, ${ }^{114}$ G. Onengut, ${ }^{114, y y}$ K. Ozdemir, ${ }^{114, z z}$ D. Sunar Cerci, ${ }^{114, \text { aaa }}$ B. Tali, ${ }^{114, \text { aaa }}$ C. Zorbilmez, ${ }^{114}$ B. Bilin, ${ }^{115}$ S. Bilmis, ${ }^{115}$ B. Isildak, ${ }^{115, \text { bbb }}$ G. Karapinar, ${ }^{115, \text { ccc }}$ M. Yalvac, ${ }^{115}$ M. Zeyrek, ${ }^{115}$ E. Gülmez, ${ }^{116}$ M. Kaya, ${ }^{116, d d d}$ O. Kaya,${ }^{116, \text { eee }}$ E. A. Yetkin, ${ }^{116, f f f}$ T. Yetkin, ${ }^{116, \text { ggg }}$ A. Cakir, ${ }^{117}$ K. Cankocak, ${ }^{117}$ S. Sen, ${ }^{117, \text { hhh }}$ F. I. Vardarllimath, ${ }^{117}$ B. Grynyov, ${ }^{118}$ L. Levchuk, ${ }^{119}$ P. Sorokin, ${ }^{119}$ R. Aggleton, ${ }^{120}$ F. Ball, ${ }^{120}$ L. Beck, ${ }^{120}$ J. J. Brooke, ${ }^{120}$ D. Burns, ${ }^{120}$ E. Clement, ${ }^{120}$ D. Cussans ${ }^{120}$ H. Flacher, ${ }^{120}$ J. Goldstein, ${ }^{120}$ M. Grimes, ${ }^{120}$ G. P. Heath ${ }^{120}$ H. F. Heath,${ }^{120}$ J. Jacob, ${ }^{120}$ L. Kreczko, ${ }^{120}$ C. Lucas, ${ }^{120}$ Z. Meng, ${ }^{120}$ D. M. Newbold, ${ }^{120, i i i}$ S. Paramesvaran, ${ }^{120}$ A. Poll, ${ }^{120}$ T. Sakuma, ${ }^{120}$ S. Seif El Nasr-storey, ${ }^{120}$ S. Senkin, ${ }^{120}$ D. Smith, ${ }^{120}$ V. J. Smith, ${ }^{120}$ K. W. Bell, ${ }^{121}$ A. Belyaev, ${ }^{121, j j j}$ C. Brew, ${ }^{121}$ R. M. Brown, ${ }^{121}$ L. Calligaris, ${ }^{121}$ D. Cieri, ${ }^{121}$ D. J. A. Cockerill, ${ }^{121}$ J. A. Coughlan, ${ }^{121}$ K. Harder, ${ }^{121}$ S. Harper,${ }^{121}$ E. Olaiya, ${ }^{121}$ D. Petyt,${ }^{121}$

C. H. Shepherd-Themistocleous, ${ }^{121}$ A. Thea, ${ }^{121}$ I. R. Tomalin, ${ }^{121}$ T. Williams, ${ }^{121}$ M. Baber,${ }^{122}$ R. Bainbridge, ${ }^{122}$ O. Buchmuller, ${ }^{122}$ A. Bundock, ${ }^{122}$ D. Burton, ${ }^{122}$ S. Casasso, ${ }^{122}$ M. Citron, ${ }^{122}$ D. Colling, ${ }^{122}$ L. Corpe ${ }^{122}$ P. Dauncey, ${ }^{122}$ G. Davies, ${ }^{122}$ A. De Wit, ${ }^{122}$ M. Della Negra, ${ }^{122}$ P. Dunne, ${ }^{122}$ A. Elwood, ${ }^{122}$ D. Futyan, ${ }^{122}$ Y. Haddad, ${ }^{122}$ G. Hall, ${ }^{122}$ G. Iles, ${ }^{122}$ R. Lane, ${ }^{122}$ C. Laner, ${ }^{122}$ R. Lucas, ${ }^{122, i i i}$ L. Lyons, ${ }^{122}$ A.-M. Magnan, ${ }^{122}$ S. Malik, ${ }^{122}$ L. Mastrolorenzo, ${ }^{122}$ J. Nash, ${ }^{122}$ A. Nikitenko, ${ }^{122, \text { uu }}$ J. Pela, ${ }^{122}$ B. Penning, ${ }^{122}$ M. Pesaresi, ${ }^{122}$ D. M. Raymond, ${ }^{122}$ A. Richards, ${ }^{122}$ A. Rose, ${ }^{122}$ C. Seez, ${ }^{122}$ A. Tapper, ${ }^{122}$ K. Uchida, ${ }^{122}$ M. Vazquez Acosta,${ }^{122, k k k}$ T. Virdee,${ }^{122, n}$ S. C. Zenz,${ }^{122}$ J. E. Cole,${ }^{123}$ P. R. Hobson, ${ }^{123}$ A. Khan, ${ }^{123}$ P. Kyberd, ${ }^{123}$ D. Leslie, ${ }^{123}$ I. D. Reid, ${ }^{123}$ P. Symonds, ${ }^{123}$ L. Teodorescu, ${ }^{123}$ M. Turner,${ }^{123}$ A. Borzou, ${ }^{124}$ K. Call, ${ }^{124}$ J. Dittmann, ${ }^{124}$ K. Hatakeyama, ${ }^{124}$ H. Liu, ${ }^{124}$ N. Pastika, ${ }^{124}$ O. Charaf, ${ }^{125}$ S. I. Cooper,${ }^{125}$ C. Henderson, ${ }^{125}$ P. Rumerio, ${ }^{125}$ D. Arcaro, ${ }^{126}$ A. Avetisyan, ${ }^{126}$ T. Bose, ${ }^{126}$ D. Gastler, ${ }^{126}$ D. Rankin, ${ }^{126}$ C. Richardson, ${ }^{126}$ J. Rohlf, ${ }^{126}$ L. Sulak, ${ }^{126}$ D. Zou, ${ }^{126}$ G. Benelli, ${ }^{127}$ E. Berry, ${ }^{127}$ D. Cutts, ${ }^{127}$ A. Ferapontov, ${ }^{127}$ A. Garabedian, ${ }^{127}$ J. Hakala,${ }^{127}$ U. Heintz, ${ }^{127}$ O. Jesus, ${ }^{127}$ E. Laird, ${ }^{127}$ G. Landsberg, ${ }^{127}$ Z. Mao, ${ }^{127}$ M. Narain, ${ }^{127}$ S. Piperov, ${ }^{127}$ S. Sagir,${ }^{127}$ E. Spencer, ${ }^{127}$ R. Syarif, ${ }^{127}$ R. Breedon, ${ }^{128}$ G. Breto, ${ }^{128}$ D. Burns, ${ }^{128}$ M. Calderon De La Barca Sanchez,${ }^{128}$ S. Chauhan, ${ }^{128}$ M. Chertok, ${ }^{128}$ J. Conway, ${ }^{128}$ R. Conway, ${ }^{128}$ P. T. Cox ${ }^{128}$ R. Erbacher,${ }^{128}$ C. Flores, ${ }^{128}$ G. Funk,${ }^{128}$ M. Gardner,${ }^{128}$ W. Ko, ${ }^{128}$ R. Lander, ${ }^{128}$ C. Mclean, ${ }^{128}$ M. Mulhearn, ${ }^{128}$ D. Pellett, ${ }^{128}$ J. Pilot, ${ }^{128}$ F. Ricci-Tam, ${ }^{128}$ S. Shalhout, ${ }^{128}$ J. Smith, ${ }^{128}$ M. Squires, ${ }^{128}$ D. Stolp, ${ }^{128}$ M. Tripathi, ${ }^{128}$ S. Wilbur, ${ }^{128}$ R. Yohay, ${ }^{128}$ R. Cousins, ${ }^{129}$ P. Everaerts, ${ }^{129}$ A. Florent, ${ }^{129}$ J. Hauser, ${ }^{129}$ M. Ignatenko, ${ }^{129}$ D. Saltzberg, ${ }^{129}$ E. Takasugi, ${ }^{129}$ V. Valuev, ${ }^{129}$ M. Weber, ${ }^{129}$ K. Burt, ${ }^{130}$ R. Clare, ${ }^{130}$ J. Ellison, ${ }^{130}$ J. W. Gary, ${ }^{130}$ G. Hanson, ${ }^{130}$ J. Heilman, ${ }^{130}$ P. Jandir, ${ }^{130}$ E. Kennedy, ${ }^{130}$ F. Lacroix,${ }^{130}$ O. R. Long, ${ }^{130}$ M. Malberti, ${ }^{130}$ M. Olmedo Negrete,${ }^{130}$ M. I. Paneva, ${ }^{130}$ A. Shrinivas, ${ }^{130}$ H. Wei, ${ }^{130}$ S. Wimpenny, ${ }^{130}$ B. R. Yates, ${ }^{130}$ J. G. Branson, ${ }^{131}$ G. B. Cerati, ${ }^{131}$ S. Cittolin, ${ }^{131}$ R. T. D’Agnolo, ${ }^{131}$ M. Derdzinski, ${ }^{131}$ R. Gerosa, ${ }^{131}$ A. Holzner, ${ }^{131}$ R. Kelley, ${ }^{131}$ D. Klein, ${ }^{131}$ J. Letts, ${ }^{131}$ I. Macneill, ${ }^{131}$ D. Olivito, ${ }^{131}$ S. Padhi,${ }^{131}$ M. Pieri, ${ }^{131}$ M. Sani, ${ }^{131}$ V. Sharma, ${ }^{131}$ S. Simon, ${ }^{131}$ M. Tadel, ${ }^{131}$ A. Vartak, ${ }^{131}$ S. Wasserbaech, ${ }^{131,111}$ C. Welke, ${ }^{131}$ J. Wood, ${ }^{131}$ F. Würthwein, ${ }^{131}$ A. Yagil,,${ }^{131}$ G. Zevi Della Porta,${ }^{131}$ R. Bhandari, ${ }^{132}$ J. Bradmiller-Feld, ${ }^{132}$ C. Campagnari, ${ }^{132}$ A. Dishaw, ${ }^{132}$ V. Dutta, ${ }^{132}$ K. Flowers, ${ }^{132}$ M. Franco Sevilla, ${ }^{132}$ P. Geffert, ${ }^{132}$ C. George, ${ }^{132}$ F. Golf, ${ }^{132}$ L. Gouskos, ${ }^{132}$ J. Gran, ${ }^{132}$ R. Heller ${ }^{132}$ J. Incandela, ${ }^{132}$ N. Mccoll, ${ }^{132}$ S. D. Mullin, ${ }^{132}$ A. Ovcharova, ${ }^{132}$ J. Richman, ${ }^{132}$ 
D. Stuart, ${ }^{132}$ I. Suarez, ${ }^{132}$ C. West,${ }^{132}$ J. Yoo, ${ }^{132}$ D. Anderson, ${ }^{133}$ A. Apresyan, ${ }^{133}$ J. Bendavid,${ }^{133}$ A. Bornheim ${ }^{133}$ J. Bunn, ${ }^{133}$ Y. Chen, ${ }^{133}$ J. Duarte, ${ }^{133}$ A. Mott,${ }^{133}$ H. B. Newman, ${ }^{133}$ C. Pena, ${ }^{133}$ M. Spiropulu, ${ }^{133}$ J. R. Vlimant,${ }^{133}$ S. Xie,${ }^{133}$ R. Y. Zhu, ${ }^{133}$ M. B. Andrews, ${ }^{134}$ V. Azzolini, ${ }^{134}$ A. Calamba, ${ }^{134}$ B. Carlson, ${ }^{134}$ T. Ferguson, ${ }^{134}$ M. Paulini, ${ }^{134}$ J. Russ,${ }^{134}$ M. Sun, ${ }^{134}$ H. Vogel, ${ }^{134}$ I. Vorobiev, ${ }^{134}$ J. P. Cumalat, ${ }^{135}$ W. T. Ford, ${ }^{135}$ F. Jensen, ${ }^{135}$ A. Johnson, ${ }^{135}$ M. Krohn, ${ }^{135}$ T. Mulholland, ${ }^{135}$ K. Stenson, ${ }^{135}$ S. R. Wagner, ${ }^{135}$ J. Alexander, ${ }^{136}$ A. Chatterjee, ${ }^{136}$ J. Chaves, ${ }^{136}$ J. Chu, ${ }^{136}$ S. Dittmer, ${ }^{136}$ N. Eggert,${ }^{136}$ N. Mirman, ${ }^{136}$ G. Nicolas Kaufman, ${ }^{136}$ J. R. Patterson, ${ }^{136}$ A. Rinkevicius, ${ }^{136}$ A. Ryd, ${ }^{136}$ L. Skinnari, ${ }^{136}$ W. Sun, ${ }^{136}$ S. M. Tan, ${ }^{136}$ Z. Tao, ${ }^{136}$ W. D. Teo, ${ }^{136}$ J. Thom, ${ }^{136}$ J. Thompson, ${ }^{136}$ J. Tucker, ${ }^{136}$ Y. Weng, ${ }^{136}$ P. Wittich, ${ }^{136}$ D. Winn, ${ }^{137}$ S. Abdullin, ${ }^{138}$ M. Albrow, ${ }^{138}$ G. Apollinari, ${ }^{138}$ S. Banerjee, ${ }^{138}$ L. A. T. Bauerdick, ${ }^{138}$ A. Beretvas, ${ }^{138}$ J. Berryhill, ${ }^{138}$ P. C. Bhat, ${ }^{138}$ G. Bolla, ${ }^{138}$ K. Burkett, ${ }^{138}$ J. N. Butler, ${ }^{138}$ H. W. K. Cheung, ${ }^{138}$ F. Chlebana, ${ }^{138}$ S. Cihangir, ${ }^{138}$ M. Cremonesi, ${ }^{138}$ V. D. Elvira, ${ }^{138}$ I. Fisk, ${ }^{138}$ J. Freeman, ${ }^{138}$ E. Gottschalk, ${ }^{138}$ L. Gray, ${ }^{138}$ D. Green, ${ }^{138}$ S. Grünendahl, ${ }^{138}$ O. Gutsche, ${ }^{138}$ D. Hare, ${ }^{138}$ R. M. Harris, ${ }^{138}$ S. Hasegawa, ${ }^{138}$ J. Hirschauer, ${ }^{138}$ Z. Hu ${ }^{138}$ B. Jayatilaka, ${ }^{138}$ S. Jindariani, ${ }^{138}$ M. Johnson, ${ }^{138}$ U. Joshi, ${ }^{138}$ B. Klima, ${ }^{138}$ B. Kreis, ${ }^{138}$ S. Lammel, ${ }^{138}$ J. Linacre, ${ }^{138}$ D. Lincoln, ${ }^{138}$ R. Lipton, ${ }^{138}$ T. Liu, ${ }^{138}$ R. Lopes De Sá, ${ }^{138}$ J. Lykken, ${ }^{138}$ K. Maeshima, ${ }^{138}$ J. M. Marraffino, ${ }^{138}$ S. Maruyama, ${ }^{138}$ D. Mason, ${ }^{138}$ P. McBride, ${ }^{138}$ P. Merkel, ${ }^{138}$ S. Mrenna, ${ }^{138}$ S. Nahn, ${ }^{138}$ C. Newman-Holmes, ${ }^{138, a}$ V. O’Dell, ${ }^{138}$ K. Pedro, ${ }^{138}$ O. Prokofyev, ${ }^{138}$ G. Rakness, ${ }^{138}$ L. Ristori, ${ }^{138}$ E. Sexton-Kennedy, ${ }^{138}$ A. Soha, ${ }^{138}$ W. J. Spalding, ${ }^{138}$ L. Spiegel,${ }^{138}$ S. Stoynev, ${ }^{138}$ N. Strobbe, ${ }^{138}$ L. Taylor, ${ }^{138}$ S. Tkaczyk, ${ }^{138}$ N. V. Tran, ${ }^{138}$ L. Uplegger, ${ }^{138}$ E. W. Vaandering, ${ }^{138}$ C. Vernieri, ${ }^{138}$ M. Verzocchi, ${ }^{138}$ R. Vidal, ${ }^{138}$ M. Wang, ${ }^{138}$ H. A. Weber ${ }^{138}$ A. Whitbeck, ${ }^{138}$ D. Acosta, ${ }^{139}$ P. Avery ${ }^{139}$ P. Bortignon, ${ }^{139}$ D. Bourilkov, ${ }^{139}$ A. Brinkerhoff,${ }^{139}$ A. Carnes, ${ }^{139}$ M. Carver ${ }^{139}$ D. Curry, ${ }^{139}$ S. Das, ${ }^{139}$ R. D. Field, ${ }^{139}$ I. K. Furic, ${ }^{139}$ J. Konigsberg, ${ }^{139}$ A. Korytov ${ }^{139}$ P. Ma, ${ }^{139}$ K. Matchev, ${ }^{139}$ H. Mei, ${ }^{139}$ P. Milenovic, ${ }^{139, m m m}$ G. Mitselmakher, ${ }^{139}$ D. Rank, ${ }^{139}$ L. Shchutska, ${ }^{139}$ D. Sperka, ${ }^{139}$ L. Thomas, ${ }^{139}$ J. Wang, ${ }^{139}$ S. Wang, ${ }^{139}$ J. Yelton, ${ }^{139}$ S. Linn, ${ }^{140}$ P. Markowitz, ${ }^{140}$ G. Martinez,${ }^{140}$ J. L. Rodriguez, ${ }^{140}$ A. Ackert, ${ }^{141}$ J. R. Adams, ${ }^{141}$ T. Adams, ${ }^{141}$ A. Askew, ${ }^{141}$ S. Bein, ${ }^{141}$ B. Diamond, ${ }^{141}$ S. Hagopian, ${ }^{141}$ V. Hagopian, ${ }^{141}$ K. F. Johnson, ${ }^{141}$ A. Khatiwada, ${ }^{141}$ H. Prosper, ${ }^{141}$ A. Santra, ${ }^{141}$ M. Weinberg, ${ }^{141}$ M. M. Baarmand, ${ }^{142}$ V. Bhopatkar, ${ }^{142}$ S. Colafranceschi, ${ }^{142, n n n}$ M. Hohlmann, ${ }^{142}$ H. Kalakhety, ${ }^{142}$ D. Noonan, ${ }^{142}$ T. Roy ${ }^{142}$ F. Yumiceva ${ }^{142}$ M. R. Adams, ${ }^{143}$ L. Apanasevich, ${ }^{143}$ D. Berry, ${ }^{143}$ R. R. Betts, ${ }^{143}$ I. Bucinskaite, ${ }^{143}$ R. Cavanaugh, ${ }^{143}$ O. Evdokimov, ${ }^{143}$ L. Gauthier, ${ }^{143}$ C. E. Gerber, ${ }^{143}$ D. J. Hofman, ${ }^{143}$ P. Kurt, ${ }^{143}$ C. O’Brien, ${ }^{143}$ I. D. Sandoval Gonzalez, ${ }^{143}$ P. Turner, ${ }^{143}$ N. Varelas, ${ }^{143}$ Z. Wu, ${ }^{143}$ M. Zakaria, ${ }^{143}$ J. Zhang, ${ }^{143}$ B. Bilki, ${ }^{144,000}$ W. Clarida, ${ }^{144}$ K. Dilsiz, ${ }^{144}$ S. Durgut, ${ }^{144}$ R. P. Gandrajula, ${ }^{144}$ M. Haytmyradov, ${ }^{144}$ V. Khristenko, ${ }^{144}$ J.-P. Merlo, ${ }^{144}$ H. Mermerkaya, ${ }^{144, p p p}$ A. Mestvirishvili, ${ }^{144}$ A. Moeller, ${ }^{144}$ J. Nachtman, ${ }^{144}$ H. Ogul, ${ }^{144}$ Y. Onel, ${ }^{144}$ F. Ozok, ${ }^{144, q q 9}$ A. Penzo, ${ }^{144}$ C. Snyder, ${ }^{144}$ E. Tiras, ${ }^{144}$ J. Wetzel, ${ }^{144}$ K. Yi, ${ }^{144}$ I. Anderson, ${ }^{145}$ B. Blumenfeld, ${ }^{145}$ A. Cocoros, ${ }^{145}$ N. Eminizer, ${ }^{145}$ D. Fehling, ${ }^{145}$ L. Feng, ${ }^{145}$ A. V. Gritsan, ${ }^{145}$ P. Maksimovic, ${ }^{145}$ M. Osherson, ${ }^{145}$ J. Roskes, ${ }^{145}$ U. Sarica, ${ }^{145}$ M. Swartz, ${ }^{145}$ M. Xiao, ${ }^{145}$ Y. Xin, ${ }^{145}$ C. You, ${ }^{145}$ A. Al-bataineh, ${ }^{146}$ P. Baringer, ${ }^{146}$ A. Bean, ${ }^{146}$ C. Bruner, ${ }^{146}$ J. Castle, ${ }^{146}$ R. P. Kenny III, ${ }^{146}$ A. Kropivnitskaya, ${ }^{146}$ D. Majumder, ${ }^{146}$ M. Malek, ${ }^{146}$ W. Mcbrayer, ${ }^{146}$ M. Murray, ${ }^{146}$ S. Sanders, ${ }^{146}$ R. Stringer, ${ }^{146}$ Q. Wang, ${ }^{146}$ A. Ivanov, ${ }^{147}$ K. Kaadze, ${ }^{147}$ S. Khalil, ${ }^{147}$ M. Makouski, ${ }^{147}$ Y. Maravin, ${ }^{147}$ A. Mohammadi, ${ }^{147}$ L. K. Saini, ${ }^{147}$ N. Skhirtladze, ${ }^{147}$ S. Toda,${ }^{147}$ D. Lange, ${ }^{148}$ F. Rebassoo, ${ }^{148}$ D. Wright, ${ }^{148}$ C. Anelli, ${ }^{149}$ A. Baden, ${ }^{149}$ O. Baron, ${ }^{149}$ A. Belloni, ${ }^{149}$ B. Calvert, ${ }^{149}$ S. C. Eno, ${ }^{149}$ C. Ferraioli, ${ }^{149}$ J. A. Gomez, ${ }^{149}$ N. J. Hadley, ${ }^{149}$ S. Jabeen, ${ }^{149}$ R. G. Kellogg, ${ }^{149}$ T. Kolberg, ${ }^{149}$ J. Kunkle, ${ }^{149}$ Y. Lu, ${ }^{149}$ A. C. Mignerey, ${ }^{149}$ Y. H. Shin, ${ }^{149}$ A. Skuja, ${ }^{149}$ M. B. Tonjes, ${ }^{149}$ S. C. Tonwar, ${ }^{149}$ A. Apyan, ${ }^{150}$ R. Barbieri, ${ }^{150}$ A. Baty, ${ }^{150}$ R. Bi,${ }^{150}$ K. Bierwagen, ${ }^{150}$ S. Brandt,${ }^{150}$ W. Busza, ${ }^{150}$ I. A. Cali ${ }^{150}$ Z. Demiragli, ${ }^{150}$ L. Di Matteo, ${ }^{150}$ G. Gomez Ceballos, ${ }^{150}$ M. Goncharov, ${ }^{150}$ D. Gulhan, ${ }^{150}$ D. Hsu, ${ }^{150}$ Y. Iiyama, ${ }^{150}$ G. M. Innocenti, ${ }^{150}$ M. Klute, ${ }^{150}$ D. Kovalskyi, ${ }^{150}$ K. Krajczar, ${ }^{150}$ Y. S. Lai, ${ }^{150}$ Y.-J. Lee, ${ }^{150}$ A. Levin, ${ }^{150}$ P. D. Luckey, ${ }^{150}$ A. C. Marini, ${ }^{150}$ C. Mcginn, ${ }^{150}$ C. Mironov, ${ }^{150}$ S. Narayanan, ${ }^{150}$ X. Niu, ${ }^{150}$ C. Paus, ${ }^{150}$ C. Roland ${ }^{150}$ G. Roland, ${ }^{150}$ J. Salfeld-Nebgen, ${ }^{150}$ G. S. F. Stephans,${ }^{150}$ K. Sumorok,${ }^{150}$ K. Tatar, ${ }^{150}$ M. Varma, ${ }^{150}$ D. Velicanu, ${ }^{150}$ J. Veverka, ${ }^{150}$ J. Wang, ${ }^{150}$ T. W. Wang, ${ }^{150}$ B. Wyslouch ${ }^{150}$ M. Yang, ${ }^{150}$ V. Zhukova, ${ }^{150}$ A. C. Benvenuti, ${ }^{151}$ B. Dahmes, ${ }^{151}$ A. Evans, ${ }^{151}$ A. Finkel, ${ }^{151}$ A. Gude, ${ }^{151}$ P. Hansen, ${ }^{151}$ S. Kalafut, ${ }^{151}$ S. C. Kao, ${ }^{151}$ K. Klapoetke, ${ }^{151}$ Y. Kubota, ${ }^{151}$ Z. Lesko, ${ }^{151}$ J. Mans, ${ }^{151}$ S. Nourbakhsh, ${ }^{151}$ N. Ruckstuhl,${ }^{151}$ R. Rusack, ${ }^{151}$ N. Tambe, ${ }^{151}$ J. Turkewitz, ${ }^{151}$ J. G. Acosta, ${ }^{152}$ S. Oliveros, ${ }^{152}$ E. Avdeeva, ${ }^{153}$ R. Bartek,${ }^{153}$ K. Bloom, ${ }^{153}$ S. Bose, ${ }^{153}$ D. R. Claes,${ }^{153}$ A. Dominguez,${ }^{153}$ C. Fangmeier ${ }^{153}$ R. Gonzalez Suarez, ${ }^{153}$ R. Kamalieddin, ${ }^{153}$ D. Knowlton, ${ }^{153}$ I. Kravchenko, ${ }^{153}$ F. Meier, ${ }^{153}$ J. Monroy, ${ }^{153}$ J. E. Siado, ${ }^{153}$ G. R. Snow, ${ }^{153}$ B. Stieger, ${ }^{153}$ M. Alyari, ${ }^{154}$ J. Dolen, ${ }^{154}$ J. George,${ }^{154}$ A. Godshalk, ${ }^{154}$ C. Harrington, ${ }^{154}$ I. Iashvili, ${ }^{154}$ J. Kaisen, ${ }^{154}$ A. Kharchilava, ${ }^{154}$ A. Kumar, ${ }^{154}$ A. Parker, ${ }^{154}$ S. Rappoccio, ${ }^{154}$ B. Roozbahani, ${ }^{154}$ G. Alverson, ${ }^{155}$ E. Barberis, ${ }^{155}$ D. Baumgartel, ${ }^{155}$ M. Chasco, ${ }^{155}$ A. Hortiangtham, ${ }^{155}$ A. Massironi, ${ }^{155}$ D. M. Morse, ${ }^{155}$ D. Nash, ${ }^{155}$ T. Orimoto, ${ }^{155}$ R. Teixeira De Lima, ${ }^{155}$ D. Trocino, ${ }^{155}$ R.-J. Wang, ${ }^{155}$ D. Wood, ${ }^{155}$ S. Bhattacharya, ${ }^{156}$ K. A. Hahn, ${ }^{156}$ 
A. Kubik, ${ }^{156}$ J. F. Low, ${ }^{156}$ N. Mucia, ${ }^{156}$ N. Odell, ${ }^{156}$ B. Pollack, ${ }^{156}$ M. H. Schmitt, ${ }^{156}$ K. Sung, ${ }^{156}$ M. Trovato, ${ }^{156}$ M. Velasco, ${ }^{156}$ N. Dev, ${ }^{157}$ M. Hildreth, ${ }^{157}$ K. Hurtado Anampa,${ }^{157}$ C. Jessop, ${ }^{157}$ D. J. Karmgard,${ }^{157}$ N. Kellams, ${ }^{157}$ K. Lannon, ${ }^{157}$ N. Marinelli, ${ }^{157}$ F. Meng, ${ }^{157}$ C. Mueller, ${ }^{157}$ Y. Musienko, ${ }^{157, j j}$ M. Planer, ${ }^{157}$ A. Reinsvold,,${ }^{157}$ R. Ruchti, ${ }^{157}$ N. Rupprecht, ${ }^{157}$ G. Smith, ${ }^{157}$ S. Taroni, ${ }^{157}$ N. Valls, ${ }^{157}$ M. Wayne, ${ }^{157}$ M. Wolf, ${ }^{157}$ A. Woodard, ${ }^{157}$ J. Alimena, ${ }^{158}$ L. Antonelli, ${ }^{158}$ J. Brinson, ${ }^{158}$ B. Bylsma, ${ }^{158}$ L. S. Durkin, ${ }^{158}$ S. Flowers, ${ }^{158}$ B. Francis, ${ }^{158}$ A. Hart, ${ }^{158}$ C. Hill, ${ }^{158}$ R. Hughes,${ }^{158}$ W. Ji, ${ }^{158}$ B. Liu, ${ }^{158}$ W. Luo, ${ }^{158}$ D. Puigh, ${ }^{158}$ M. Rodenburg, ${ }^{158}$ B. L. Winer,${ }^{158}$ H. W. Wulsin, ${ }^{158}$ O. Driga,${ }^{159}$ P. Elmer, ${ }^{159}$ J. Hardenbrook ${ }^{159}$ P. Hebda ${ }^{159}$ D. Marlow, ${ }^{159}$ T. Medvedeva, ${ }^{159}$ M. Mooney, ${ }^{159}$ J. Olsen, ${ }^{159}$ C. Palmer,${ }^{159}$ P. Piroué, ${ }^{159}$ D. Stickland, ${ }^{159}$ C. Tully, ${ }^{159}$ A. Zuranski,${ }^{159}$ S. Malik, ${ }^{160}$ A. Barker, ${ }^{161}$ V. E. Barnes,${ }^{161}$ D. Benedetti, ${ }^{161}$ S. Folgueras, ${ }^{161}$ L. Gutay, ${ }^{161}$ M. K. Jha, ${ }^{161}$ M. Jones, ${ }^{161}$ A. W. Jung, ${ }^{161}$ K. Jung, ${ }^{161}$ D. H. Miller ${ }^{161}$ N. Neumeister, ${ }^{161}$ B. C. Radburn-Smith, ${ }^{161}$ X. Shi,${ }^{161}$ J. Sun, ${ }^{161}$ A. Svyatkovskiy, ${ }^{161}$ F. Wang,${ }^{161}$ W. Xie, ${ }^{161}$ L. Xu ${ }^{161}$ N. Parashar, ${ }^{162}$ J. Stupak, ${ }^{162}$ A. Adair, ${ }^{163}$ B. Akgun, ${ }^{163}$ Z. Chen, ${ }^{163}$ K. M. Ecklund, ${ }^{163}$ F. J. M. Geurts, ${ }^{163}$ M. Guilbaud, ${ }^{163}$ W. Li, ${ }^{163}$ B. Michlin, ${ }^{163}$ M. Northup, ${ }^{163}$ B. P. Padley, ${ }^{163}$ R. Redjimi, ${ }^{163}$ J. Roberts, ${ }^{163}$ J. Rorie,${ }^{163}$ Z. Tu, ${ }^{163}$ J. Zabel, ${ }^{163}$ B. Betchart, ${ }^{164}$ A. Bodek, ${ }^{164}$ P. de Barbaro, ${ }^{164}$ R. Demina, ${ }^{164}$ Y. t. Duh, ${ }^{164}$ Y. Eshaq, ${ }^{164}$ T. Ferbel, ${ }^{164}$ M. Galanti,${ }^{164}$ A. Garcia-Bellido, ${ }^{164}$ J. Han, ${ }^{164}$ O. Hindrichs, ${ }^{164}$ A. Khukhunaishvili, ${ }^{164}$ K. H. Lo, ${ }^{164}$ P. Tan, ${ }^{164}$ M. Verzetti, ${ }^{164}$ J. P. Chou, ${ }^{165}$

E. Contreras-Campana, ${ }^{165}$ Y. Gershtein, ${ }^{165}$ T. A. Gómez Espinosa, ${ }^{165}$ E. Halkiadakis, ${ }^{165}$ M. Heindl, ${ }^{165}$ D. Hidas, ${ }^{165}$ E. Hughes, ${ }^{165}$ S. Kaplan, ${ }^{165}$ R. Kunnawalkam Elayavalli, ${ }^{165}$ S. Kyriacou, ${ }^{165}$ A. Lath, ${ }^{165}$ K. Nash, ${ }^{165}$ H. Saka, ${ }^{165}$ S. Salur, ${ }^{165}$ S. Schnetzer, ${ }^{165}$ D. Sheffield, ${ }^{165}$ S. Somalwar, ${ }^{165}$ R. Stone, ${ }^{165}$ S. Thomas, ${ }^{165}$ P. Thomassen, ${ }^{165}$ M. Walker, ${ }^{165}$ M. Foerster, ${ }^{166}$ J. Heideman, ${ }^{166}$ G. Riley, ${ }^{166}$ K. Rose, ${ }^{166}$ S. Spanier, ${ }^{166}$ K. Thapa ${ }^{166}$ O. Bouhali, ${ }^{167, \text { rrr }}$ A. Castaneda Hernandez, ${ }^{167, \text { rrr }}$ A. Celik, ${ }^{167}$ M. Dalchenko, ${ }^{167}$ M. De Mattia ${ }^{167}$ A. Delgado, ${ }^{167}$ S. Dildick, ${ }^{167}$ R. Eusebi, ${ }^{167}$ W. Flanagan, ${ }^{167}$ J. Gilmore, ${ }^{167}$ T. Huang, ${ }^{167}$ E. Juska, ${ }^{167}$ T. Kamon, ${ }^{167, \text { sss }}$ V. Krutelyov, ${ }^{167}$ R. Mueller, ${ }^{167}$ Y. Pakhotin, ${ }^{167}$ R. Patel, ${ }^{167}$ A. Perloff, ${ }^{167}$ L. Perniè, ${ }^{167}$ D. Rathjens, ${ }^{167}$ A. Rose, ${ }^{167}$ A. Safonov, ${ }^{167}$ A. Tatarinov, ${ }^{167}$ K. A. Ulmer, ${ }^{167}$ N. Akchurin, ${ }^{168}$ C. Cowden, ${ }^{168}$ J. Damgov, ${ }^{168}$ C. Dragoiu, ${ }^{168}$ P. R. Dudero, ${ }^{168}$ J. Faulkner, ${ }^{168}$ S. Kunori, ${ }^{168}$ K. Lamichhane, ${ }^{168}$ S. W. Lee, ${ }^{168}$ T. Libeiro, ${ }^{168}$ S. Undleeb, ${ }^{168}$ I. Volobouev, ${ }^{168}$ Z. Wang, ${ }^{168}$ A. G. Delannoy, ${ }^{169}$ S. Greene, ${ }^{169}$ A. Gurrola, ${ }^{169}$ R. Janjam, ${ }^{169}$ W. Johns,${ }^{169}$ C. Maguire, ${ }^{169}$ A. Melo, ${ }^{169}$ H. Ni, ${ }^{169}$ P. Sheldon, ${ }^{169}$ S. Tuo, ${ }^{169}$ J. Velkovska, ${ }^{169}$ Q. Xu, ${ }^{169}$ M. W. Arenton, ${ }^{170}$ P. Barria, ${ }^{170}$ B. Cox, ${ }^{170}$ J. Goodell, ${ }^{170}$ R. Hirosky, ${ }^{170}$ A. Ledovskoy, ${ }^{170} \mathrm{H}$. Li,${ }^{170}$ C. Neu, ${ }^{170}$ T. Sinthuprasith,,${ }^{170}$ X. Sun, ${ }^{170}$ Y. Wang, ${ }^{170}$ E. Wolfe,${ }^{170}$ F. Xia ${ }^{170}$ C. Clarke, ${ }^{171}$ R. Harr, ${ }^{171}$ P. E. Karchin,${ }^{171}$ C. Kottachchi Kankanamge Don, ${ }^{171}$ P. Lamichhane, ${ }^{171}$ J. Sturdy, ${ }^{171}$ D. A. Belknap, ${ }^{172}$ S. Dasu, ${ }^{172}$ L. Dodd ${ }^{172}$ S. Duric, ${ }^{172}$ B. Gomber, ${ }^{172}$ M. Grothe,${ }^{172}$ M. Herndon,,${ }^{172}$ A. Hervé, ${ }^{172}$ P. Klabbers, ${ }^{172}$ A. Lanaro, ${ }^{172}$ A. Levine,${ }^{172}$ K. Long, ${ }^{172}$ R. Loveless, ${ }^{172}$ I. Ojalvo, ${ }^{172}$ T. Perry, ${ }^{172}$ G. A. Pierro, ${ }^{172}$ G. Polese, ${ }^{172}$ T. Ruggles, ${ }^{172}$ A. Savin, ${ }^{172}$ A. Sharma, ${ }^{172}$ N. Smith, ${ }^{172}$ W. H. Smith, ${ }^{172}$ D. Taylor, ${ }^{172}$ P. Verwilligen, ${ }^{172}$ and N. Woods ${ }^{172}$

(CMS Collaboration)

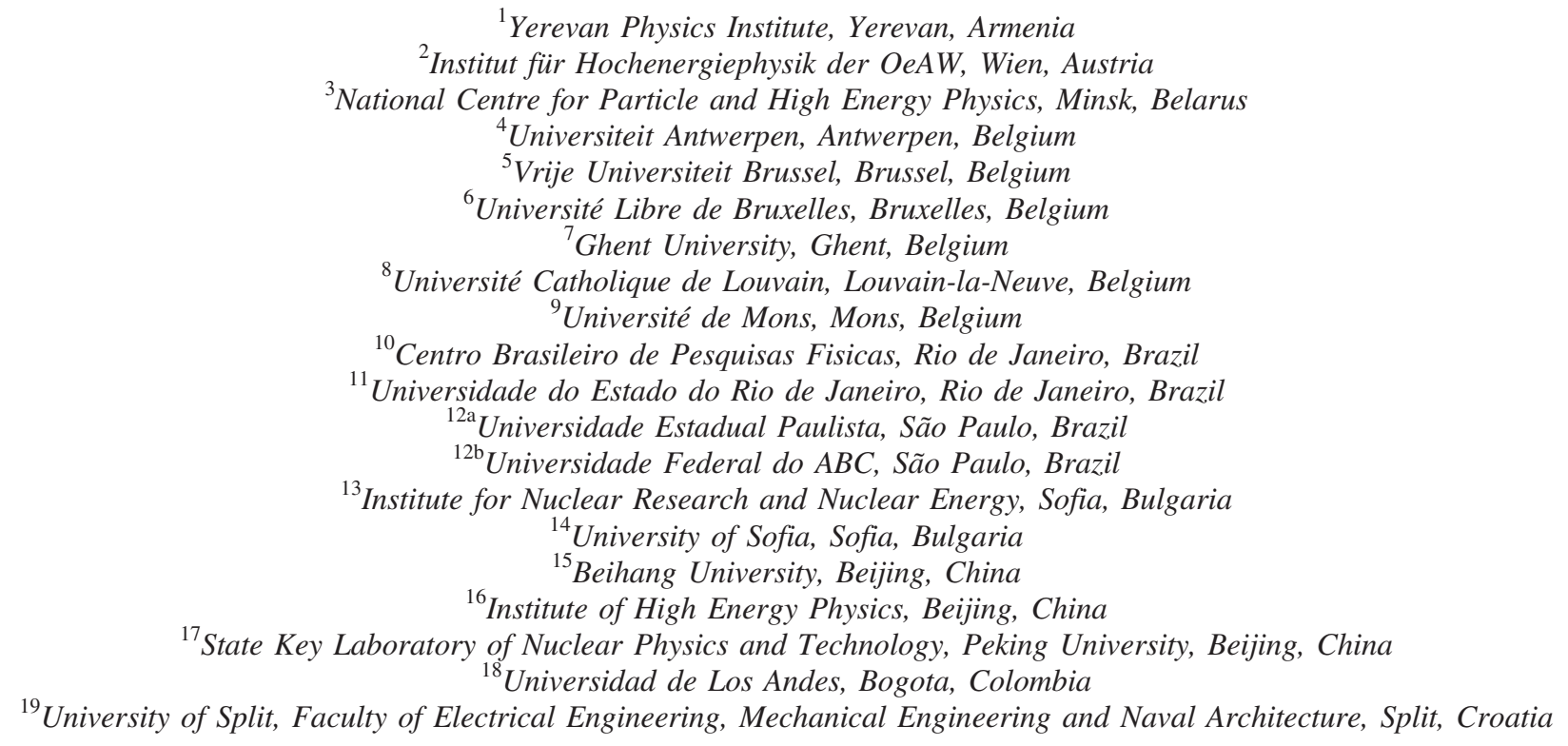


${ }^{20}$ University of Split, Faculty of Science, Split, Croatia

${ }^{21}$ Institute Rudjer Boskovic, Zagreb, Croatia

${ }^{22}$ University of Cyprus, Nicosia, Cyprus

${ }^{23}$ Charles University, Prague, Czech Republic

${ }^{24}$ Universidad San Francisco de Quito, Quito, Ecuador

${ }^{25}$ Academy of Scientific Research and Technology of the Arab Republic of Egypt,

Egyptian Network of High Energy Physics, Cairo, Egypt

${ }^{26}$ National Institute of Chemical Physics and Biophysics, Tallinn, Estonia

${ }^{27}$ Department of Physics, University of Helsinki, Helsinki, Finland

${ }^{28}$ Helsinki Institute of Physics, Helsinki, Finland

${ }^{29}$ Lappeenranta University of Technology, Lappeenranta, Finland

${ }^{30}$ DSM/IRFU, CEA/Saclay, Gif-sur-Yvette, France

${ }^{31}$ Laboratoire Leprince-Ringuet, Ecole Polytechnique, IN2P3-CNRS, Palaiseau, France

${ }^{32}$ Institut Pluridisciplinaire Hubert Curien, Université de Strasbourg, Université de Haute Alsace Mulhouse, CNRS/IN2P3, Strasbourg, France

${ }^{33}$ Centre de Calcul de l'Institut National de Physique Nucleaire et de Physique des Particules, CNRS/IN2P3, Villeurbanne, France

${ }^{34}$ Université de Lyon, Université Claude Bernard Lyon 1, CNRS-IN2P3, Institut de Physique Nucléaire de Lyon, Villeurbanne, France

${ }^{35}$ Georgian Technical University, Tbilisi, Georgia

${ }^{36}$ Tbilisi State University, Tbilisi, Georgia

${ }^{37}$ RWTH Aachen University, I. Physikalisches Institut, Aachen, Germany

${ }^{38}$ RWTH Aachen University, III. Physikalisches Institut A, Aachen, Germany

${ }^{39}$ RWTH Aachen University, III. Physikalisches Institut B, Aachen, Germany

${ }^{40}$ Deutsches Elektronen-Synchrotron, Hamburg, Germany

${ }^{41}$ University of Hamburg, Hamburg, Germany

${ }^{42}$ Institut für Experimentelle Kernphysik, Karlsruhe, Germany

${ }^{43}$ Institute of Nuclear and Particle Physics (INPP), NCSR Demokritos, Aghia Paraskevi, Greece

${ }^{44}$ National and Kapodistrian University of Athens, Athens, Greece

${ }^{45}$ University of Ioánnina, Ioánnina, Greece

${ }^{46}$ MTA-ELTE Lendület CMS Particle and Nuclear Physics Group, Eötvös Loránd University

${ }^{47}$ Wigner Research Centre for Physics, Budapest, Hungary

${ }^{48}$ Institute of Nuclear Research ATOMKI, Debrecen, Hungary

${ }^{49}$ University of Debrecen, Debrecen, Hungary

${ }^{50}$ National Institute of Science Education and Research, Bhubaneswar, India

${ }^{51}$ Panjab University, Chandigarh, India

${ }^{52}$ University of Delhi, Delhi, India

${ }^{53}$ Saha Institute of Nuclear Physics, Kolkata, India

${ }^{54}$ Indian Institute of Technology Madras, Madras, India

${ }^{55}$ Bhabha Atomic Research Centre, Mumbai, India

${ }^{56}$ Tata Institute of Fundamental Research, Mumbai, India

${ }^{57}$ Indian Institute of Science Education and Research (IISER), Pune, India

${ }^{58}$ Institute for Research in Fundamental Sciences (IPM), Tehran, Iran

${ }^{59}$ University College Dublin, Dublin, Ireland

${ }^{60 \mathrm{a}}$ INFN Sezione di Bari, Bari, Italy

${ }^{60 \mathrm{~b}}$ Università di Bari, Bari, Italy

${ }^{60 c}$ Politecnico di Bari, Bari, Italy

${ }^{61 \mathrm{a}}$ INFN Sezione di Bologna, Bologna, Italy

${ }^{61 \mathrm{~b}}$ Università di Bologna, Bologna, Italy

${ }^{62 a}$ INFN Sezione di Catania, Catania, Italy

${ }^{62 \mathrm{~b}}$ Università di Catania, Catania, Italy

${ }^{63 \mathrm{a}}$ INFN Sezione di Firenze, Firenze, Italy

${ }^{63 \mathrm{~b}}$ Università di Firenze, Firenze, Italy

${ }^{64}$ INFN Laboratori Nazionali di Frascati, Frascati, Italy

${ }^{65 a}$ INFN Sezione di Genova, Genova, Italy

${ }^{65 \mathrm{~b}}$ Università di Genova, Genova, Italy

${ }^{66 a}$ INFN Sezione di Milano-Bicocca, Milano, Italy

${ }^{66 \mathrm{~b}}$ Università di Milano-Bicocca, Milano, Italy

${ }^{67 a}$ INFN Sezione di Napoli, Roma, Italy

${ }^{67 \mathrm{~b}}$ Università di Napoli 'Federico II', Roma, Italy

${ }^{67 \mathrm{c}}$ Università della Basilicata, Roma, Italy

${ }^{67 d}$ Università G. Marconi, Roma, Italy 
${ }^{68 \mathrm{a}}$ INFN Sezione di Padova, Trento, Italy

${ }^{68 \mathrm{~b}}$ Università di Padova, Trento, Italy

${ }^{68 \mathrm{c}}$ Università di Trento, Trento, Italy

${ }^{69 a}$ INFN Sezione di Pavia, Pavia, Italy

${ }^{69 \mathrm{~b}}$ Università di Pavia, Pavia, Italy

${ }^{70 a}$ INFN Sezione di Perugia, Perugia, Italy

${ }^{70 \mathrm{~b}}$ Università di Perugia, Perugia, Italy

${ }^{71 a}$ INFN Sezione di Pisa, Pisa, Italy

${ }^{71 \mathrm{~b}}$ Università di Pisa, Pisa, Italy

${ }^{71 \mathrm{c} S c u o l a}$ Normale Superiore di Pisa, Pisa, Italy

${ }^{72 a}$ INFN Sezione di Roma, Pisa, Italy

${ }^{72 \mathrm{~b}}$ Università di Roma, Pisa, Italy

${ }^{73 a}$ INFN Sezione di Torino, Novara, Italy

${ }^{73 b}$ Università di Torino, Novara, Italy

${ }^{73 \mathrm{c}}$ Università del Piemonte Orientale, Novara, Italy

${ }^{74 a}$ INFN Sezione di Trieste, Trieste, Italy

${ }^{74 \mathrm{~b}}$ Università di Trieste, Trieste, Italy

${ }^{75}$ Kyungpook National University, Daegu, Korea

${ }^{76}$ Chonbuk National University, Jeonju, Korea

${ }^{77}$ Hanyang University, Seoul, Korea

${ }^{78}$ Korea University, Seoul, Korea

${ }^{79}$ Seoul National University, Seoul, Korea

${ }^{80}$ University of Seoul, Seoul, Korea

${ }^{81}$ Sungkyunkwan University, Suwon, Korea

${ }^{82}$ Vilnius University, Vilnius, Lithuania

${ }^{83}$ National Centre for Particle Physics, Universiti Malaya, Kuala Lumpur, Malaysia

${ }^{84}$ Centro de Investigacion y de Estudios Avanzados del IPN, Mexico City, Mexico

${ }^{85}$ Universidad Iberoamericana, Mexico City, Mexico

${ }^{86}$ Benemerita Universidad Autonoma de Puebla, Puebla, Mexico

${ }^{87}$ Universidad Autónoma de San Luis Potosí, San Luis Potosí, Mexico

${ }^{88}$ University of Auckland, Auckland, New Zealand

${ }^{89}$ University of Canterbury, Christchurch, New Zealand

${ }^{90}$ National Centre for Physics, Quaid-I-Azam University, Islamabad, Pakistan

${ }^{91}$ National Centre for Nuclear Research, Swierk, Poland

${ }^{92}$ Institute of Experimental Physics, Faculty of Physics, University of Warsaw, Warsaw, Poland

${ }^{93}$ Laboratório de Instrumentação e Física Experimental de Partículas, Lisboa, Portugal

${ }^{94}$ Joint Institute for Nuclear Research, Dubna, Russia

${ }^{95}$ Petersburg Nuclear Physics Institute, Gatchina (St. Petersburg), Russia

${ }^{96}$ Institute for Nuclear Research, Moscow, Russia

${ }^{97}$ Institute for Theoretical and Experimental Physics, Moscow, Russia

${ }^{98}$ National Research Nuclear University 'Moscow Engineering Physics Institute' (MEPhI), Moscow, Russia

${ }^{99}$ P.N. Lebedev Physical Institute, Moscow, Russia

${ }^{100}$ Skobeltsyn Institute of Nuclear Physics, Lomonosov Moscow State University, Moscow, Russia

${ }^{101}$ State Research Center of Russian Federation, Institute for High Energy Physics, Protvino, Russia

${ }^{102}$ University of Belgrade, Faculty of Physics and Vinca Institute of Nuclear Sciences, Belgrade, Serbia

${ }^{103}$ Centro de Investigaciones Energéticas Medioambientales y Tecnológicas (CIEMAT), Madrid, Spain

${ }^{104}$ Universidad Autónoma de Madrid, Madrid, Spain

${ }^{105}$ Universidad de Oviedo, Oviedo, Spain

${ }^{106}$ Instituto de Física de Cantabria (IFCA), CSIC-Universidad de Cantabria, Santander, Spain

${ }^{107}$ CERN, European Organization for Nuclear Research, Geneva, Switzerland

${ }^{108}$ Paul Scherrer Institut, Villigen, Switzerland

${ }^{109}$ Institute for Particle Physics, ETH Zurich, Zurich, Switzerland

${ }^{110}$ Universität Zürich, Zurich, Switzerland

${ }^{111}$ National Central University, Chung-Li, Taiwan

${ }^{112}$ National Taiwan University (NTU), Taipei, Taiwan

${ }^{113}$ Chulalongkorn University, Faculty of Science, Department of Physics, Bangkok, Thailand

${ }^{114}$ Cukurova University, Adana, Turkey

${ }^{115}$ Middle East Technical University, Physics Department, Ankara, Turkey

${ }^{116}$ Bogazici University, Istanbul, Turkey

${ }^{117}$ Istanbul Technical University, Istanbul, Turkey 
${ }^{118}$ Institute for Scintillation Materials of National Academy of Science of Ukraine, Kharkov, Ukraine

${ }^{119}$ National Scientific Center, Kharkov Institute of Physics and Technology, Kharkov, Ukraine

${ }^{120}$ University of Bristol, Bristol, United Kingdom

${ }^{121}$ Rutherford Appleton Laboratory, Didcot, United Kingdom

${ }^{122}$ Imperial College, London, United Kingdom

${ }^{123}$ Brunel University, Uxbridge, United Kingdom

${ }^{124}$ Baylor University, Waco, USA

${ }^{125}$ The University of Alabama, Tuscaloosa, USA

${ }^{126}$ Boston University, Boston, USA

${ }^{127}$ Brown University, Providence, USA

${ }^{128}$ University of California, Davis, Davis, USA

${ }^{129}$ University of California, Los Angeles, USA

${ }^{130}$ University of California, Riverside, Riverside, USA

${ }^{131}$ University of California, San Diego, La Jolla, USA

${ }^{132}$ University of California, Santa Barbara, Santa Barbara, USA

${ }^{133}$ California Institute of Technology, Pasadena, USA

${ }^{134}$ Carnegie Mellon University, Pittsburgh, USA

${ }^{135}$ University of Colorado Boulder, Boulder, USA

${ }^{136}$ Cornell University, Ithaca, USA

${ }^{137}$ Fairfield University, Fairfield, USA

${ }^{138}$ Fermi National Accelerator Laboratory, Batavia, USA

${ }^{139}$ University of Florida, Gainesville, USA

${ }^{140}$ Florida International University, Miami, USA

${ }^{141}$ Florida State University, Tallahassee, USA

${ }^{142}$ Florida Institute of Technology, Melbourne, USA

${ }^{143}$ University of Illinois at Chicago (UIC), Chicago, USA

${ }^{144}$ The University of Iowa, Iowa City, USA

${ }^{145}$ Johns Hopkins University, Baltimore, USA

${ }^{146}$ The University of Kansas, Lawrence, USA

${ }^{147}$ Kansas State University, Manhattan, USA

${ }^{148}$ Lawrence Livermore National Laboratory, Livermore, USA

${ }^{149}$ University of Maryland, College Park, USA

${ }^{150}$ Massachusetts Institute of Technology, Cambridge, USA

${ }^{151}$ University of Minnesota, Minneapolis, USA

${ }^{152}$ University of Mississippi, Oxford, USA

${ }^{153}$ University of Nebraska-Lincoln, Lincoln, USA

${ }^{154}$ State University of New York at Buffalo, Buffalo, USA

${ }^{155}$ Northeastern University, Boston, USA

${ }^{156}$ Northwestern University, Evanston, USA

${ }^{157}$ University of Notre Dame, Notre Dame, USA

${ }^{158}$ The Ohio State University, Columbus, USA

${ }^{159}$ Princeton University, Princeton, USA

${ }^{160}$ University of Puerto Rico, Mayaguez, USA

${ }^{161}$ Purdue University, West Lafayette, USA

${ }^{162}$ Purdue University Calumet, Hammond, USA

${ }^{163}$ Rice University, Houston, USA

${ }^{164}$ University of Rochester, Rochester, USA

${ }^{165}$ Rutgers, The State University of New Jersey, Piscataway, USA

${ }^{166}$ University of Tennessee, Knoxville, USA

${ }^{167}$ Texas A\&M University, College Station, USA

${ }^{168}$ Texas Tech University, Lubbock, USA

${ }^{169}$ Vanderbilt University, Nashville, USA

${ }^{170}$ University of Virginia, Charlottesville, USA

${ }^{171}$ Wayne State University, Detroit, USA

${ }^{172}$ University of Wisconsin-Madison, Madison, WI, USA

${ }^{\mathrm{a}}$ Deceased.

${ }^{\mathrm{b}}$ Also at Vienna University of Technology, Vienna, Austria.

${ }^{\mathrm{c}}$ Also at State Key Laboratory of Nuclear Physics and Technology, Peking University, Beijing, China. 
${ }^{\mathrm{d}}$ Also at Institut Pluridisciplinaire Hubert Curien, Université de Strasbourg, Université de Haute Alsace Mulhouse, CNRS/IN2P3, Strasbourg, France.

${ }^{\mathrm{e}}$ Also at Universidade Estadual de Campinas, Campinas, Brazil.

${ }^{\mathrm{f}}$ Also at Centre National de la Recherche Scientifique (CNRS) - IN2P3, Paris, France.

${ }^{\mathrm{g}}$ Also at Université Libre de Bruxelles, Bruxelles, Belgium.

${ }^{\mathrm{h}}$ Also at Deutsches Elektronen-Synchrotron, Hamburg, Germany.

${ }^{\mathrm{i}}$ Also at Joint Institute for Nuclear Research, Dubna, Russia.

${ }^{\mathrm{j}}$ Also at British University in Egypt, Cairo, Egypt.

${ }^{\mathrm{k}}$ Also at Zewail City of Science and Technology, Zewail, Egypt.

${ }^{1}$ Also at Ain Shams University, Cairo, Egypt.

${ }^{\mathrm{m}}$ Also at Université de Haute Alsace, Mulhouse, France.

${ }^{\mathrm{n}}$ Also at CERN, European Organization for Nuclear Research, Geneva, Switzerland.

${ }^{\circ}$ Also at Skobeltsyn Institute of Nuclear Physics, Lomonosov Moscow State University, Moscow, Russia.

${ }^{\mathrm{p}}$ Also at Tbilisi State University, Tbilisi, Georgia.

${ }^{\mathrm{q}}$ Also at RWTH Aachen University, III. Physikalisches Institut A, Aachen, Germany.

${ }^{\mathrm{r}}$ Also at University of Hamburg, Hamburg, Germany.

${ }^{\mathrm{s}}$ Also at Brandenburg University of Technology, Cottbus, Germany.

${ }^{t}$ Also at Institute of Nuclear Research ATOMKI, Debrecen, Hungary.

"Also at MTA-ELTE Lendület CMS Particle and Nuclear Physics Group, Eötvös Loránd University.

${ }^{v}$ Also at University of Debrecen, Debrecen, Hungary.

${ }^{w}$ Also at Indian Institute of Science Education and Research, Bhopal, India.

${ }^{\mathrm{x}}$ Also at University of Visva-Bharati, Santiniketan, India.

${ }^{y}$ Also at King Abdulaziz University, Jeddah, Saudi Arabia.

${ }^{\mathrm{z}}$ Also at University of Ruhuna, Matara, Sri Lanka.

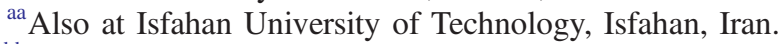

${ }^{\mathrm{bb}}$ Also at University of Tehran, Department of Engineering Science, Tehran, Iran.

${ }^{\mathrm{cc}}$ Also at Plasma Physics Research Center, Science and Research Branch, Islamic Azad University, Tehran, Iran.

${ }^{\mathrm{dd}}$ Also at Università degli Studi di Siena, Siena, Italy.

${ }^{e e}$ Also at Purdue University, West Lafayette, USA.

${ }^{\mathrm{ff}}$ Also at International Islamic University of Malaysia, Kuala Lumpur, Malaysia.

${ }^{\mathrm{gg}}$ Also at Malaysian Nuclear Agency, MOSTI, Kajang, Malaysia.

${ }^{\text {hh }}$ Also at Consejo Nacional de Ciencia y Tecnología, Mexico city, Mexico.

${ }^{\text {ii }}$ Also at Warsaw University of Technology, Institute of Electronic Systems, Warsaw, Poland.

${ }^{\mathrm{jj}}$ Also at Institute for Nuclear Research, Moscow, Russia.

${ }^{k k}$ Also at National Research Nuclear University 'Moscow Engineering Physics Institute' (MEPhI), Moscow, Russia.

${ }^{11}$ Also at St. Petersburg State Polytechnical University, St. Petersburg, Russia.

${ }^{\mathrm{mm}}$ Also at University of Florida, Gainesville, USA.

${ }^{\mathrm{nn}}$ Also at California Institute of Technology, Pasadena, USA.

${ }^{\circ 0}$ Also at Faculty of Physics, University of Belgrade, Belgrade, Serbia.

${ }^{\mathrm{pp}}$ Also at INFN Sezione di Roma, Università di Roma, Roma, Italy.

${ }^{\mathrm{qq}}$ Also at National Technical University of Athens, Athens, Greece.

${ }^{\text {rr }}$ Also at Scuola Normale e Sezione dell'INFN, Pisa, Italy.

${ }^{\mathrm{ss}}$ Also at National and Kapodistrian University of Athens, Athens, Greece.

"Also at Riga Technical University, Riga, Latvia.

${ }^{\mathrm{uu}}$ Also at Institute for Theoretical and Experimental Physics, Moscow, Russia.

${ }^{v v}$ Also at Albert Einstein Center for Fundamental Physics, Bern, Switzerland.

${ }^{w w}$ Also at Gaziosmanpasa University, Tokat, Turkey.

${ }^{\mathrm{xx}}$ Also at Mersin University, Mersin, Turkey.

${ }^{y y}$ Also at Cag University, Mersin, Turkey.

${ }^{\mathrm{zz}}$ Also at Piri Reis University, Istanbul, Turkey.

${ }^{a a a}$ Also at Adiyaman University, Adiyaman, Turkey.

${ }^{\mathrm{bbb}}$ Also at Ozyegin University, Istanbul, Turkey.

${ }^{c c c}$ Also at Izmir Institute of Technology, Izmir, Turkey.

ddd Also at Marmara University, Istanbul, Turkey.

${ }^{e e e}$ Also at Kafkas University, Kars, Turkey.

${ }^{\text {fff }}$ Also at Istanbul Bilgi University, Istanbul, Turkey.

${ }^{\text {ggg }}$ Also at Yildiz Technical University, Istanbul, Turkey.

hhh Also at Hacettepe University, Ankara, Turkey.

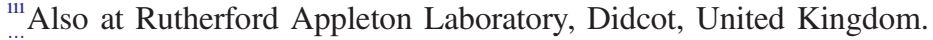

${ }^{\mathrm{ijj}}$ Also at School of Physics and Astronomy, University of Southampton, Southampton, United Kingdom. 


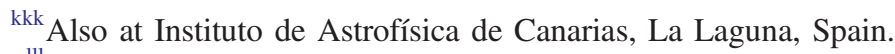

${ }^{111}$ Also at Utah Valley University, Orem, USA.

${ }^{\mathrm{mmm}}$ Also at University of Belgrade, Faculty of Physics and Vinca Institute of Nuclear Sciences, Belgrade, Serbia.

${ }^{n n n}$ Also at Facoltà Ingegneria, Università di Roma, Roma, Italy.

${ }^{000}$ Also at Argonne National Laboratory, Argonne, USA.

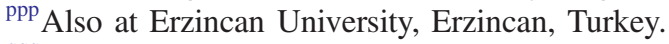

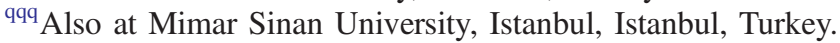

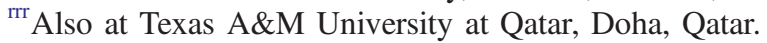

${ }^{\text {sss }}$ Also at Kyungpook National University, Daegu, Korea. 